Article

\title{
Diversity Variation of Silica-Scaled Chrysophytes Related to Differences in Physicochemical Variables in Estuaries of Rivers in an Arctic Watershed
}

\author{
Anna Bessudova ${ }^{1, *(D)}$, Viktor Gabyshev ${ }^{2}\left(\mathbb{D}\right.$, Alena Firsova $^{1} \mathbb{D}$, Olga Gabysheva $^{2}$, Yurij Bukin ${ }^{1}(\mathbb{D}$ \\ and Yelena Likhoshway ${ }^{1}$ (D)
}

1 Limnological Institute Siberian Branch of the Russian Academy of Sciences, 3 Ulan-Batorskaya, 664033 Irkutsk, Russia; adfir71@yandex.ru (A.F.); bukinyura@mail.ru (Y.B.); likhoshway@mail.ru (Y.L.)

2 Institute for Biological Problems of Cryolithozone, Siberian Branch of the Russian Academy of Science, 41 Lenina Avenue, 677980 Yakutsk, Russia; v.a.gabyshev@yandex.ru (V.G.); g89248693006@yandex.ru (O.G.)

* Correspondence: annabessudova@mail.ru; Tel.: +7-964-22-68-893

\section{check for}

updates

Citation: Bessudova, A.; Gabyshev, V.; Firsova, A.; Gabysheva, O.; Bukin, Y.; Likhoshway, Y. Diversity Variation of Silica-Scaled Chrysophytes Related to Differences in Physicochemical Variables in Estuaries of Rivers in an Arctic Watershed. Sustainability 2021, 13, 13768. https://doi.org/10.3390/ su132413768

Academic Editor: Sushanta Kumar Saha

Received: 10 November 2021 Accepted: 6 December 2021 Published: 13 December 2021

Publisher's Note: MDPI stays neutral with regard to jurisdictional claims in published maps and institutional affiliations.

Copyright: (c) 2021 by the authors. Licensee MDPI, Basel, Switzerland. This article is an open access article distributed under the terms and conditions of the Creative Commons Attribution (CC BY) license (https:/ / creativecommons.org/licenses/by/ $4.0 /)$.

\begin{abstract}
The present study examined the taxonomic diversity of silica-scaled chrysophytes in the estuaries of the Arctic watershed of Yakutia in the context of global climate change, as these aquatic organisms are highly sensitive to environmental changes. Previously, 41 species of silica-scaled chrysophytes were recorded in the waters of Yakutia. In the present study, we supplemented this list with 55 species. We observed a high species richness (82 taxa) of silica-scaled chrysophytes in the study area. Of these, eight species were recorded in the waters of Russia for the first time. At present, the study area represents the northernmost habitat for most species observed during the study. The diversity of silica-scaled chrysophytes in Arctic rivers is comparable to or even greater than the diversity of chrysophycean flora at the middle latitudes. Most of these chrysophytes are represented by polyzonal and ubiquitous species. During 2008-2010, we noted increased relative numbers of boreal species in northern waters compared with data obtained during the previous 30 years. Overall, the species richness of silica-scaled chrysophytes in the studied rivers increased with increase in water transparency, temperature, and magnesium ion concentration, but decreased with increase in surfactant concentration. The origin of samples from a certain river; distance of the sampling site from the estuary; water temperature, transparency, and colour; and concentration of carbon dioxide, magnesium ions, total iron, surfactants, and oil products affect the species composition of silica-scaled chrysophytes in the study area. Our findings are fundamental to evaluate the current status of Arctic aquatic microflora and its further monitoring in the context of anthropogenic and climatic impacts.
\end{abstract}

Keywords: Chrysophyceae; floristics; species richness; species composition; environment; biogeography; Arctic; Yakutia

\section{Introduction}

Representatives of the class Chrysophyceae have long been recognised as important indicators of environmental conditions [1-3]. They inhabit all climatic zones, including the tropics [4-7] and the Arctic [8-10]. However, their diversity and abundance are the highest at the middle latitudes [11-13], decreasing northward [14]. Few studies have explored the diversity of silica-scaled chrysophytes in northern polar waters $\left(\sim 66^{\circ} 33^{\prime} \mathrm{N}\right)$. To date, 4 species in a small lake in Alaska [15], 17 species in the lakes of the Mackenzie Delta in Canada [16], 21 species in the Disko Island and Søndre Strømfjord region of West Greenland [17], 5 species in Central Norway [18], 32 species in Swedish Lapland [19,20], 6 species in Finnish Lapland [21], 75 species in the Bolshezemelskaya and Vorkuta tundra in Russia [22,23], 42 species in the Polar Urals of Russia [24,25], 44 species in the gulfs and bays of the Kara Sea in Russia [26,27], 25 species in the Khantay Reservoir in Russia [28], 
26 species in the waters of the Taymyr Peninsula in Russia [8,9], and 8 species in the estuary of the Anabar River, Yakutia, Russia [10] have been recorded.

In the face of the current trends of global climate change [29,30] and rising atmospheric $\mathrm{CO}_{2}$ concentrations [31-33], silica-scaled chrysophytes at latitudes must be studied, considering that these organisms are highly sensitive to environmental changes. During the 20th century, the mean annual temperature of the Earth's surface increased by $0.6{ }^{\circ} \mathrm{C}$. In the Arctic, the temperature has risen to almost two times the earlier value and is expected to further increase by $4-7^{\circ} \mathrm{C}$ in the present century [34]. The duration of open-water period, that is, the duration of the growing season [35], is an important factor that relies upon ambient temperature [35]. From 1980 to 2014, the duration of open water period in various water bodies in Asian Russia was extended by 4.63 to 11 days on average every decade [36], and this trend has been predicted to persist in the coming decades [34]. This prolongation of growing season favours the northward dispersal of southern species, leading to the expansion of their biogeographical ranges [37-39]. In a previous study [40], we analysed factors affecting the abundance and diversity of chrysophyceans in northern waters and hypothesised a scenario of their dispersal. Recent studies have frequently reported increased abundance of silica-scaled chrysophytes in boreal and Arctic lakes [29,30]. In their study in the Subarctic Region, Siver et al. [22] confirmed this trend for the first time based on the high diversity (75 species) of silica-scaled chrysophytes in lakes and ponds in the Bolshezemelskaya and Vorkuta tundra; the authors recorded some representatives of boreal flora, such as species of the genus Lepidochromonas (Lepidochromonas homolepis (Preisig \& D.J. Hibberd) Kapustin \& Guiry, Lepidochromonas quadrispina (Thomsen \& Kristiansen) Kapustin \& Guiry, Lepidochromonas subquadrangularis (Preisig \& D.J. Hibberd) Kapustin \& Guiry, and Lepidochromonas undulata (Preisig \& D.J. Hibberd) Kapustin \& Guiry) [22], at higher latitudes for the first time.

The water bodies we investigated in the present study are located north of the Arctic Circle $\left(68^{\circ}-72^{\circ}\right.$ parallel) in the Arctic zone. Samples collected from this region remain relatively understudied, particularly using electron microscopy. In previous studies using light microscopy, 32 species of silica-scaled chrysophytes were identified in the waters of Yakutia, including 21 species in the Lena River delta [41-47]. However, these records warrant a taxonomic revision given the limitations of light microscopy. Subsequent studies using electron microscopy revealed 41 species in the waters of lakes Labynkyr and Vorota [48], lake Toko and its surrounding area, and the estuary of Anabar [10].

Given water temperature is a factor determining the diversity of silica-scaled chrysophytes in northern reservoirs, we aimed to establish the biogeographic structures of communities of these organisms in the rivers of the Arctic region of Yakutia and compare these structures with those in other previously studied northern reservoirs. In this study, we tested the hypothesis of the introduction of species from temperate latitudes into Arctic reservoirs in the context of global climate change.

\section{Site Description}

\subsection{Habitat Characteristics}

The republic of Sakha (Yakutia) is the largest region in Russia (Figure 1), covering a total area of approximately $31,03,200 \mathrm{~km}^{2}$. Over $40 \%$ of Yakutia lies north of the Arctic Circle. The climate is extremely continental, with long winters and short summers [49].

The study sites are located north of the Polar Circle downstream the rivers, which flow in the Arctic geographic zone over three terrestrial ecosystems, including tundra, wooded tundra, and taiga. The southern boundary of the tundra in the region runs west-to-east along the right shores of the Bur River (left tributary of the Olenyok River), crossing the Lena River in the north of Kyusyur.

It runs parallel to the Indigirka River for up to $70 \mathrm{~km}$ and then turns southward toward the Kolyma River, crossing it near Nizhnekolymsk. The study area is situated in the permafrost zone. 


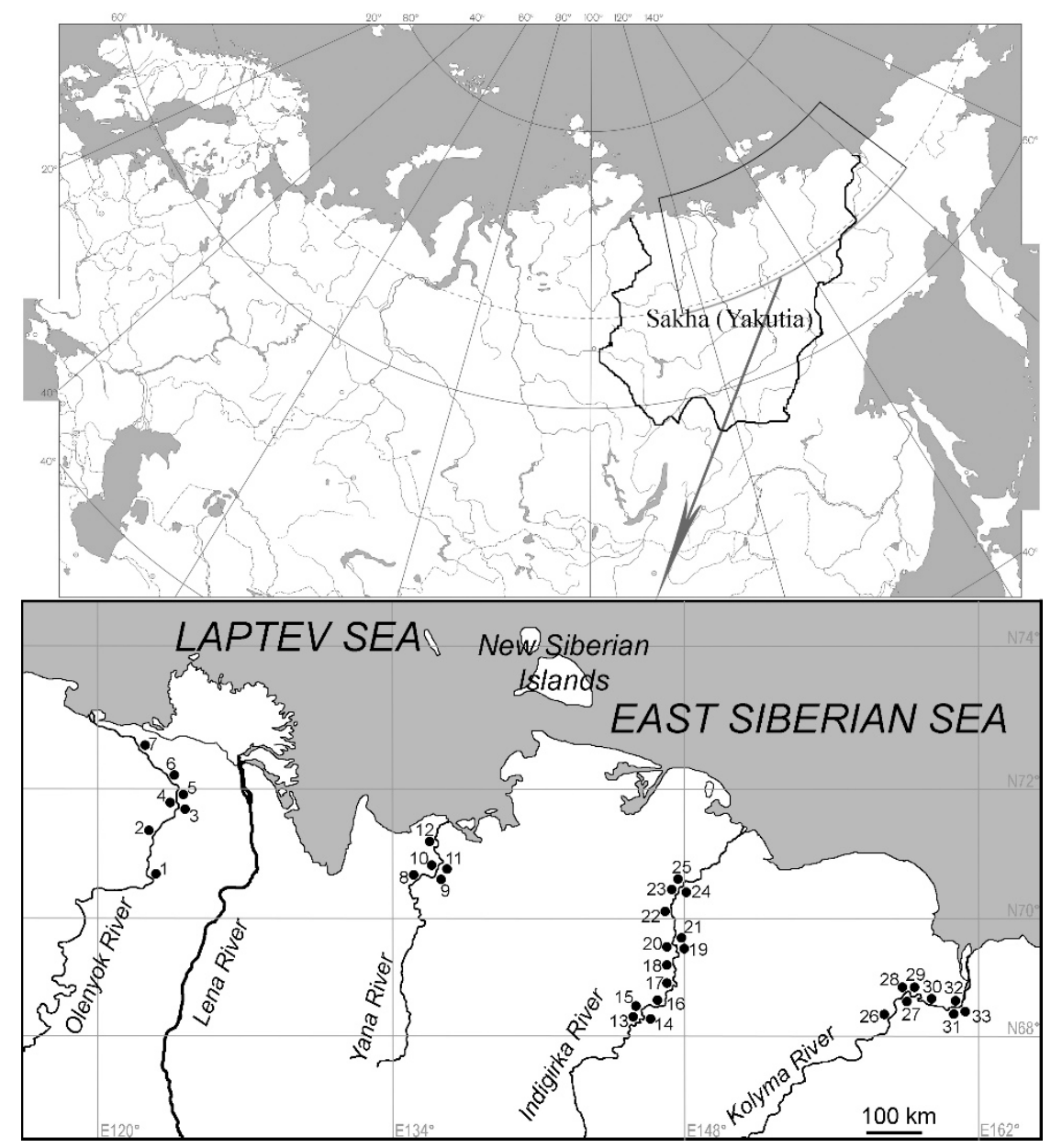

Figure 1. Schematic map of the study region and locations of sampling sites. Broken line marks the boundary of the Arctic Circle.

Olenyok is one of the largest rivers, where the anthropogenic load is low. Amongst rivers in Asia, Olenyok is the fifteenth longest $(2270 \mathrm{~km})$ and the nineteenth largest by the area of watershed $\left(219,000 \mathrm{~km}^{2}\right)$ [50]. There are only two small settlements without industrial facilities, namely Olenyok and Taymylyr, on the banks of this large river. The river has never known navigation. Indigirka is a large river of the Arctic watershed in East Siberia; it is $1900 \mathrm{~km}$ long, and its drainage basin expands over $362,000 \mathrm{~km}^{2}$. There are almost no human activities along the river, and its ecosystems remain pristine. Yana is another large river of the Arctic watershed in East Siberia; it is $872 \mathrm{~km}$ long, and its drainage basin expands over $230,000 \mathrm{~km}^{2}$. The river begins at the confluence of the rivers Sartang and Dulgalakh. As the river flows into the Laptev Sea, it forms a huge river delta spanning 10,200 $\mathrm{km}^{2}$ [51]. Kolyma is a large river of the Arctic watershed in East Siberia; it is $2600 \mathrm{~km}$ long, and its drainage basin expands over $665,000 \mathrm{~km}^{2}$ [50]. Large fields of mineral resources are found in the drainage basins of these rivers; therefore, intense development of these areas is anticipated in near future. Mineral resources of Yana, Indigirka, and Kolyma are being developed since the second half of last century. The Kolyma Hydroelectric Station regulates the flow of the Kolyma River. The study sites are situated far from large scientific centres and remain hardly accessible. There is no developed route network in the study area, and the mode of transportation is mainly by air; hence, the field samples were collected by rafting.

\subsection{Climate}

The climate of the region is under a great influence of the Siberian High, which develops in winter at the centre of Asia; a large offset of this high covers the entirety 
of East Siberia. The climate is also strongly affected by frequent air intrusions from the Arctic Ocean, which are poor in aqueous vapour in summer. According to the data on WorldClim (www.worldclim.org), the mean annual ambient temperature at the sampling sites varies between -12.4 and $-14.8^{\circ} \mathrm{C}$, with the maximum temperature recorded in summer $\left(14.9-18.6^{\circ} \mathrm{C}\right)$; mean annual precipitation is $160-256 \mathrm{~mm}$. The mean duration of open water, which determines the length of the growing season, is 115, 118, 122, and 135 days in Olenyok, Yana, Indigirka, and Kolyma, respectively [52,53]. Based on our observations in summer when the water flow was low, the median water temperature of the rivers was $14.8^{\circ} \mathrm{C}$.

\section{Materials and Methods}

\subsection{Field and Laboratory Methods}

Samples were collected on 10-14 August 2008, 26 June-2 July 2009, 17-18 July 2009, and 5-7 August 2010, either from the littoral zone or from the midstream in the surface water layer (0-0.3 m) of Olenyok, Yana, Indigirka, and Kolyma, respectively, (see Figure 1).

Phytoplankton samples $(n=33)$ were collected using the Apstein net $[54,55]$ with a SEFAR NITEX filter cloth ( $30 \mu \mathrm{m}$ mesh). The collected material was fixed in $4 \%$ formaldehyde solution at the 1:10 ratio.

Transmission electron microscopy was used for the identification of chrysophytes based on the structure of their scales. Samples were transferred to $1.5 \mathrm{~mL}$ Eppendorf tubes and centrifuged using the MiniSpin centrifuge (Eppendorf, Germany) at 13,400 rpm for $10 \mathrm{~min}$. The supernatant was removed using a pipette, and deionised water was added to the precipitate. The procedure was performed three times. Next, $30 \% \mathrm{H}_{2} \mathrm{O}_{2}$ was added to the precipitate, and the sample was heated in a thermostat at $80{ }^{\circ} \mathrm{C}$ for $5 \mathrm{~h}$. Thereafter, the precipitate was washed with deionised water and centrifuged. The procedure was performed five times. The washed samples were pipetted onto a grid (diameter, $3 \mathrm{~mm}$ ) covered with Formvar support, dried at room temperature, and examined under a transmission electron microscope (LEO 906E; Carl Zeiss, Germany).

Chemical analyses of 25 water samples were performed following standard methods [56,57]. Gaseous components of water $\left(\mathrm{O}_{2}\right.$ and $\left.\mathrm{CO}_{2}\right)$ and specific physical parameters were measured in situ, and the remaining chemical analyses were performed in vitro. A Secchi disc was used to measure water transparency. Water colour was determined using a photometric method with the SF-26 tool. $\mathrm{pH}$ was measured using a potentiometric method with the Multitest IPL-101 device. A gravimetric method was used to determine the suspended solid content [57]. Oxygen and carbon dioxide concentrations were measured using a titration method with iodimetric determination and phenolphthalein, respectively. Oxygen saturation was determined using a calculation method, and the hardness of water was measured using a titration method. Water salinity was calculated as the sum of ions using the following methods: turbidimetry for sulphate anions; flame spectrophotometry for potassium and sodium cations; mercurimetry for chloride ions; and titration for calcium, magnesium, and bicarbonate ions. A photometric method with the SF-26 tool was applied to determine nutrient concentrations. Nessler's reagent, Griess reagent, salicylic acid, ammonium molybdate, and sulfosalicylic acid were used for the measurement of ammonium ion, nitrite ion, nitrate ion, phosphate and silicon ions, and total iron, respectively. A combined reagent composed of ammonium molybdate and ascorbic acid was used to determine total phosphorus content. Chemical oxygen demand (COD) was measured with the Fluorat-02 device using a luminescent method. A titration method with iodimetric determination was used to measure biological oxygen demand (BOD). A fluorimetric method with the Fluorat-02 device was used to measure the levels of oil products, phenols, and anionic surfactants. The maximum allowable concentrations (MACs) in the waters of commercial fishing [58] are used in this article. 


\subsection{Geographical Distribution}

To determine eventual changes in the context of global climate change, we analysed the geographical distribution of silica-scaled chrysophytes in waters located north of the Arctic Circle over the long-term. Considering the scarcity of such investigations, with no studies conducted in some regions since 1981, we undertook preliminary revision of species composition to match the current taxonomy of silica-scaled chrysophytes (Table S1). We ascribed the taxa of silica-scaled chrysophytes to the formerly proposed latitudinal and longitudinal groups of geographical distribution $[59,60]$ according to their preferred distribution in a given geographic zone and their geographic range over the continents. The following types of geographic distribution were included within the latitudinal group: P: polyzonal (species found in all climatic zones); A-Bor: Arctic-boreal (species found in the Northern Hemisphere in the temperate and/or Arctic zones); Bor: boreal (species only found in the boreal zone); and Arc: Arctic (species only found in the Arctic zone). The following types of geographic distribution were included within the longitudinal group: $\mathrm{K}$ : cosmopolitan (species found on all six continents); W: widespread (species absent from one or two continents); R: species with scattered distribution (species rarely found across different latitudes); BP: bipolar species (species occurring at the middle latitudes of the Northern and Southern Hemispheres); and End: endemic (species restricted to a certain geographical range in a continent). We used the tag 'unknown' i.e., with unknown geographical characteristic) for new species, bipolar species, or species identified only to the genus level. Since some species from the literature we analysed were no longer valid, species composition in Table S1 is presented following the taxonomic revision. Species complexes within the groups Paraphysomonas vestita (Stokes) De Saedeeler and Paraphysomonas imperforata Lucas as well as Synura petersenii f. kufferathii Petersen \& Hansen, which could not be synonymised, are simply designated as species.

\subsection{Statistical Analysis}

Only those samples for which all physicochemical parameters were known, and which harboured at least one chrysophycean species, were included in the statistical analysis $(n=18)$.

PERMANOVA [61] in the R package 'Vegan' [62] was used for analysing the effects of abiotic physical and chemical parameters on the species composition of chrysophytes. Species composition data in samples were represented as a binary table, with rows and columns describing samples and species, respectively. The presence of a species in a sample was coded as 1 and its absence as 0 . The Jaccard distance matrix was calculated for PERMANOVA based on the table of species composition. $p$ values were calculated based on 10,000 permutations. The effect of a factor was assumed true when the $p$ value was $\leq 0.05$. Furthermore, variance coefficients $\left(R^{2}\right)$ were obtained using PERMANOVA, which represented the extent of variation in dependent variables (differences in species composition) driven by the independent variables.

Differences in species composition amongst samples based on the Jaccard distances were visualised using non-metric multidimensional scaling (NMDS). Gradient vectors of abiotic physical and chemical parameters that truly affected (according to PERMANOVA) the species composition of samples were plotted on an NMDS biplot. Additionally, a gradient vector of the species richness of samples was plotted on the NMDS biplot. All analyses were performed in R using the 'Vegan' [62] package following the tutorial [63].

\subsection{Data Availability}

All raw data, including the binary table of species per sample and physicochemical indicators of water per sample, are provided in Table S2. 


\section{Results}

\subsection{Diversity and Geographic Distribution of Chrysophytes}

In total, 82 species belonging to six genera, namely Chrysosphaerella $(n=5)$, Paraphysomonas $(n=12)$, Lepidochromonas $(n=10)$, Spiniferomonas $(n=10)$, Mallomonas $(n=20)$, and Synura $(n=25)$ (Table 1, Figures S1-S7), were identified in the estuaries of Olenyok, Yana, Indigirka, and Kolyma. Of these, eight species, including Lepidochromonas eiffelii, Lepidochromonas elegantissima, Lepidochromonas subquadrangularis, Mallomonas lychenensis, Synura cf. americana, Synura cf. cornuta, Synura soroconopea, and Synura cf. vinlandica, were described in the waters of Russia for the first time. No silica-scaled chrysophytes were detected in samples collected from stations 10 (estuary of Yana), 13, 15-19, 22-25 (estuaries of Indigirka), 28, 29, and 33 (estuaries of Kolyma). Species composition differed amongst the four rivers.

Table 1. List of chrysophytes and their distribution in the estuaries. Site numbers correspond to those in Figure 1 and Table 1. Species found in Russia for the first time are in bold.

\begin{tabular}{|c|c|c|c|c|c|c|c|c|c|c|c|c|c|c|c|c|c|c|c|c|}
\hline No & Site & 1 & 2 & 3 & 4 & 5 & 6 & 7 & 8 & 9 & 11 & 12 & 14 & 20 & 21 & 26 & 27 & 30 & 31 & 32 \\
\hline 1. & Chrysosphaerella brevispina Korshikov & & & & + & + & + & & & & + & + & & & & & + & & & + \\
\hline 2. & $\begin{array}{c}\text { Ch. coronacircumspina Wujek \& Kristiansen } \\
\text { in Wujek, Gretz \& Wujek }\end{array}$ & + & + & + & + & + & + & + & & & & + & & & & & & & & \\
\hline 3. & Ch. longispina Lauterborn & & & & + & + & & & & & & & & & & & & & & \\
\hline 4. & Ch. rotundata Škaloudová \& Škaloud & & & & & + & & & & & & & & & & & & & & \\
\hline 5. & Chrysosphaerella sp. & & & & + & + & & & & & & + & & & & & & & & + \\
\hline
\end{tabular}

6. Paraphysomonas acuminata acuminata Scoble \& Cavalier-Smith

\begin{tabular}{|c|c|c|c|c|c|c|c|c|}
\hline 7. & P. bandaiensis Takahashi & & & + & & & & \\
\hline 8. & P. circumvallata Preisig \& Hibberd & + & + & + & & & & \\
\hline 9. & P. gladiata Preisig \& Hibberd & ++ & - & ++ & & + & & \\
\hline 10. & P. punctata Preisig \& Hibberd & & . & +++ & & & & \\
\hline 11. & $\begin{array}{l}\text { P. cf. punctata ssp. simplicior Preisig \& } \\
\text { Hibberd }\end{array}$ & & & + & & + & & \\
\hline 12. & P. vulgaris Scoble \& Cavalier-Smith & + & & + & & & & + \\
\hline 13. & $\begin{array}{l}\text { P. uniformis hemiradia Scoble \& } \\
\text { Cavalier-Smith }\end{array}$ & + & + & $+\quad+$ & + & + & $+\quad+$ & \\
\hline 14. & Paraphysomonas sp. 1 & & ++ & ++ & & & & \\
\hline 15. & Paraphysomonas sp. 2 & & & + & & & & \\
\hline 16. & Paraphysomonas sp. 3 & & ++ & + & & & & \\
\hline 17. & Paraphysomonas sp. 4 & & & + & & & & \\
\hline 18. & $\begin{array}{l}\text { Lepidochromonas butcheri (Pennick \& Clarke) } \\
\text { Kapustin \& Guiry }\end{array}$ & & & + & & & & \\
\hline 19. & $\begin{array}{l}\text { L. coronata (Moestrup \& Zimmerman) } \\
\text { Kapustin \& Guiry }\end{array}$ & & & + & & & & \\
\hline 20. & L. diadernifera (Takahashi) Kristiansen & & & + & & & & \\
\hline 21. & L. eiffelii (Thomsen) Kapustin \& Guiry & & & + & & & & \\
\hline 22. & $\begin{array}{l}\text { L. elegantissima (Kling \& Kristiansen) } \\
\text { Kapustin \& Guiry }\end{array}$ & & & + & & & & \\
\hline 23. & $\begin{array}{c}\text { L. quadrispina (Thomsen \& Kristiansen) } \\
\text { Kapustin \& Guiry }\end{array}$ & & & + & & & & \\
\hline
\end{tabular}


Table 1. Cont.

\begin{tabular}{|c|c|c|c|c|c|c|c|c|c|c|c|c|c|c|c|c|c|c|c|}
\hline No & $\begin{array}{ll}\text { Number Species } & \text { Site } \\
\end{array}$ & 1 & 2 & 3 & 4 & 5 & 6 & 8 & 9 & 11 & 12 & 14 & 20 & 21 & 26 & 27 & 30 & 31 & 32 \\
\hline 24. & $\begin{array}{l}\text { L. quadrispina (Preisig \& Hibberd) Kapustin } \\
\text { \& Guiry }\end{array}$ & & & & & + & & & & & & & & & & & & & \\
\hline 25. & $\begin{array}{l}\text { L. undulata (Preisig \& Hibberd) Kapustin \& } \\
\text { Guiry }\end{array}$ & & & & & + & & & & & & & & & & & & & \\
\hline 26. & Lepidochromonas sp. 1 & & & & & + & & & & & & & & & & & & & \\
\hline 27. & Lepidochromonas sp. 2 & & & & & + & & & & & & & & & & & & & \\
\hline 28. & Spiniferomonas abei Takahashi & & & & & + & & & & & & & & & & & & & \\
\hline 29. & S. bourrellyi Takahashi & & + & & + & + & + & & & & + & & & & & & & & \\
\hline 30. & S. conica Takahashi & & + & & & & & & & & & & & & & & & & \\
\hline 31. & S. cornuta Balonov & & + & & & + & + & & & & + & & & & & & & & \\
\hline 32. & S. minuta Nicholls & & & & + & & & & & & & & & & & & & & \\
\hline 33. & S. serrata Nicholls & & + & + & + & + & & + & & & + & & & & & & & & + \\
\hline 34. & S. silverensis Nicholls & & + & & & & & & & & & & & & & & & & \\
\hline 35. & S. takahashii Nicholls & & & & & + & & & & & & & & & & & & & \\
\hline 36. & S. trioralis Takahashi & & & + & + & + & ++ & + & & & + & & & & + & & & & \\
\hline 37. & S. trioralis f. cuspidata Balonov & + & & & + & + & & & & & & & & & & & & & \\
\hline 38. & Mallomonas acaroides Perty & & & + & + & & + & & + & + & + & & & & + & + & & & + \\
\hline 39. & M. actinoloma Takahashi & & & & & & & & & + & + & & & & & & & & \\
\hline 40. & M. akrokomos Ruttner & & & + & + & + & + & & & & + & & & & & & & + & \\
\hline 41. & M. alpina Pascher \& Ruttner & + & + & + & + & + & ++ & + & + & + & + & & & & + & + & & + & + \\
\hline
\end{tabular}

42. M. alata $\mathrm{f}$. hualvensis Asmund, Cronberg \& Dürrschmidt

\begin{tabular}{|c|c|c|c|c|c|c|c|c|c|c|c|c|c|c|c|c|c|c|c|c|}
\hline 43. & M. areolata Nygaard & & + & & & & & & & + & & & & & & & & & & \\
\hline 44. & M. caudata Iwanoff & & & + & + & & & & & & & & & & & + & + & & + & + \\
\hline 45. & M. cratis Harris \& Bradley & & & + & & & & & & & & & & & & & & & & \\
\hline 46. & M. crassisquama (Asmund) Fott & + & + & + & + & + & & + & & + & + & + & + & & & + & + & & + & + \\
\hline 47. & M. elongata Reverdin & & & & & & & & & & & + & & & & + & + & & & + \\
\hline 48. & M. eoa Takahashi in Asmund \& Takahashi & & & & & + & & & & & & & & & & & & & & \\
\hline 49. & M. kuzminii Gusev \& Kulikovskiy & & + & + & & & + & & & + & + & & & & & & & & & + \\
\hline 50. & M. lychenensis Conrad & & & & + & & & & & & & & & & & & & & & \\
\hline 51. & M. multiunca Asmund & & + & & & & & & & & & & & & & & & & & \\
\hline 52. & M. papillosa Harris \& Bradley & & & & + & & & & & & & & & & & & & & & \\
\hline 53. & M. parvula Dürrschmidt & & & & & & & & & & + & & & & & & & & & \\
\hline 54. & M. cf. pumilio Harris \& Bradley & & & & + & & & & & & & & & & & & & & & \\
\hline 55. & M. punctifera Korshikov & & & + & + & + & & & & + & + & + & & & & & & & & \\
\hline 56. & M. striata Asmund & & & & & & & & & & & & & & & + & & & & \\
\hline 57. & M. tonsurata Teiling & + & + & + & + & + & + & + & & + & + & + & + & & & + & + & & & + \\
\hline 58. & Synura cf. americana Kynclová \& Škaloud & + & + & + & + & + & + & + & + & + & + & + & + & + & & + & + & + & + & + \\
\hline 59. & S. borealis Škaloud \& Škaloudová & + & & + & + & + & + & + & + & + & + & + & & + & + & + & + & & & + \\
\hline 60. & S. cf. cornuta Škaloud, Škaloudová \& Siver & + & & + & & & & & & & & & & & & & & & & \\
\hline 61. & S. conopea Kynclová \& Škaloud & + & + & + & + & + & + & + & + & + & + & + & & & & + & & + & & + \\
\hline
\end{tabular}


Table 1. Cont.

\begin{tabular}{|c|c|c|c|c|c|c|c|c|c|c|c|c|c|c|c|c|c|c|c|c|}
\hline No & Site & 1 & 2 & 3 & 4 & 56 & 6 & 7 & 8 & 9 & 11 & 12 & 14 & 20 & 21 & 26 & 27 & 30 & 31 & 32 \\
\hline 62. & S. curtispina (Petersen \& Hansen) Asmund & & & & & + & & & & & & + & & & & & & & & \\
\hline 63. & S. curtispina f. reticulata Asmund & & & + & + & + & & & & & + & + & & & & & & & & \\
\hline 64. & S. echinulate Korshikov & & + & & + & + & & + & & + & + & & & & & & + & & & + \\
\hline 65. & S. glabra (Korshikov) Škaloud \& Kynclová & + & & & + & + & + & + & & & & + & + & & & & & & & + \\
\hline 66. & $\begin{array}{l}\text { S. leptorhabda Nicholls in Nicholls \& } \\
\text { Gerrath }\end{array}$ & & & & & & & & & & & + & & & & & & & & \\
\hline 67. & S. macropora Škaloud \& Kynclová & + & . & + & & & & & & & + & + & & & & & & & & + \\
\hline 68. & S. mammillosa Takahashi & & & & + & & & & & + & + & + & & & & & & & & + \\
\hline 69. & $\begin{array}{l}\text { S. nygaardii (Petersen \& Hansen) } \\
\text { Kristiansen }\end{array}$ & & & + & & & & & & & + & & & & & & & & + & \\
\hline 70. & $\begin{array}{l}\text { S. petersenii (Korshikov) Škaloud \& } \\
\text { Kynclová }\end{array}$ & + & & + & + & & + & + & & & + & & & + & & & + & & + & \\
\hline 71. & S. petersenii f. taymyrensis Kristiansen & & & & + & & & & & & & & & & & & & & & \\
\hline 72. & $\begin{array}{c}\text { S. praefracta (Asmund) Škaloud \& } \\
\text { Škaloudová }\end{array}$ & + & + & + & + & ++ & + & ++ & + & & + & + & + & & & + & + & + & + & + \\
\hline 73. & S. cf. soroconopea Jo, Shin, Kim \& Siver & + & & + & + & + & & ++ & + & + & + & + & & & & & + & + & & + \\
\hline 74. & S. sphagnicola Korshikov & & & & + & + & & + & & & & & & & & & & & & + \\
\hline 75. & S. spinosa Korshikov & & & + & & + & & + & & & + & & & & & + & + & + & & + \\
\hline 76. & S. spinosa f. longispina Petersen \& Hansen & & + & + & & & & + & & & + & & & & & + & & + & + & + \\
\hline 77. & S. uvella Ehrenberg & & & & & & & & & & & & & & & + & & + & & \\
\hline 78. & $\begin{array}{l}\text { S. cf. vinlandica Škaloud, Škaloudová \& } \\
\text { Siver }\end{array}$ & & & & & & & + & & & & & & & & & & & & \\
\hline 79. & Synura sp. 1 & & & & & & & & & & & + & & & & & & & & \\
\hline 80. & Synura sp. 2 & & & & + & & + & + & & + & & & & & & & + & & & + \\
\hline 81. & Synura sp. 3 & & & & + & & & & & & & & & & & & & & & \\
\hline \multirow[t]{2}{*}{82.} & Synura sp. 4 & & & + & + & + & & ++ & + & & + & + & & & & & & & & \\
\hline & Total & 14 & 22 & 26 & 40 & 522 & 21 & 216 & 6 & 15 & 23 & 31 & 5 & 3 & 1 & 16 & 16 & 7 & 9 & 24 \\
\hline
\end{tabular}

The highest diversity of chrysophytes was recorded in Olenyok, with 75 species belonging to Chrysosphaerella $(n=5)$, Paraphysomonas $(n=12)$, Lepidochromonas $(n=10)$, Spiniferomonas $(n=10)$, Mallomonas $(n=16)$ and Synura (22) (Table 2). Of these, the cosmopolitan species Chrysosphaerella coronacircumspina, Paraphysomonas gladiata, Spiniferomonas bourrellyi, Spiniferomonas serrata, Spiniferomonas trioralis, Mallomonas akrokomos, Mallomonas crassisquama and Synura echinulate were the most abundant and most frequently detected at the study site. Station 1 differed from the other stations in the estuary in terms of hydrochemical parameters. At station 1 , total ion content was $270.14 \mathrm{mg} \cdot \mathrm{L}^{-1}$, and total phosphorus content was $186 \mu \mathrm{g} \cdot \mathrm{L}^{-1}$ (Table 2). However, the species richness at station 1 (14 species) was lower than that at the other stations situated downstream. Further downstream in the north toward station 5 , species composition became more diverse and species richness was the maximum (52 species). Amongst all stations in Olenyok, stations 4 and 5, located $\sim 3 \mathrm{~km}$ apart, harboured the most diverse species composition. The observed differences in the species composition and hydrochemical parameters across stations of the same river can be explained by the effects of the Bur River, which flows close to station 4 . At station 4, total ion content was $67.46 \mathrm{mg} \cdot \mathrm{L}^{-1}$ and $\mathrm{pH}$ was 6.78 ; meanwhile, at station 5 , these values were, respectively, $180.67 \mathrm{mg} \cdot \mathrm{L}^{-1}$ and 7.52 . Moreover, six species, namely Paraphysomonas acuminata, Spiniferomonas minuta, Mallomonas lychenensis, Mallomonas papil- 
losa, Mallomonas cf. pumilio, and Synura sp. 3, were detected only at station 4. Meanwhile, 36 species were detected only at station 5 . Furthermore, station 5 harboured high richness of species belonging to the genera Paraphysomonas and Lepidochromonas, which are atypical in the waters at high latitudes. Specifically, Paraphysomonas cf. punctata ssp. simplicior, Paraphysomonas vulgaris, Lepidochromonas eiffelii, and Lepidochromonas elegantissima, which are typical of the waters at middle latitudes, were identified in the Arctic Region for the first time. Water temperature at station $5\left(16.8^{\circ} \mathrm{C}\right)$ is favourable for the development of boreal species. Closer to the estuary, water temperature falls to $15^{\circ} \mathrm{C}$, drastically reducing species richness (21 species).

Table 2. A listing of the sampling sites from the rivers of Yakutia area included in this study. Site names and physicochemical parameters of the river mouths (site numbers in Figure 1).

\begin{tabular}{|c|c|c|c|c|c|c|c|c|c|c|c|c|c|c|}
\hline $\begin{array}{l}\text { Site } \\
\text { Num- } \\
\text { ber }\end{array}$ & $\underset{\text { Date }}{\text { Sampling }}$ & Coordinates & River & $\mathrm{T},{ }^{\circ} \mathrm{C}$ & $\underset{\text { Epi }}{\mathrm{pH}}$ & $\begin{array}{l}\text { Water Trans- } \\
\text { parency, } \\
\text { m }\end{array}$ & $\begin{array}{l}\text { Suspended } \\
\text { Matter, } \\
\text { mg/L }\end{array}$ & $\begin{array}{c}\Sigma \\
\text { Ions, } \\
\text { mg/L }\end{array}$ & $\underset{\mathrm{mg} / \mathrm{L}}{\mathrm{Si}}$ & $\begin{array}{l}P_{\text {total }} \\
\mu \mathrm{g} / \mathrm{L}\end{array}$ & $\begin{array}{l}\mathrm{O}_{2} \\
\mathrm{mg} / \mathrm{L}\end{array}$ & $\begin{array}{l}\mathrm{CO}_{2}, \\
\mathrm{mg} / \mathrm{L}\end{array}$ & $\begin{array}{l}\text { Number } \\
\text { of } \\
\text { Species }\end{array}$ & $\begin{array}{c}\text { Total } \\
\text { Number } \\
\text { of Species } \\
\text { in the } \\
\text { River }\end{array}$ \\
\hline 1 & $\begin{array}{c}10 \\
\text { August } \\
2008\end{array}$ & $\begin{array}{l}70^{\circ} 62^{\prime} 12^{\prime \prime} \mathrm{N}, \\
122^{\circ} 55^{\prime} 654^{\prime \prime} \mathrm{E}\end{array}$ & Olenyok & 16.0 & 7.89 & 4.0 & 7.20 & 270.14 & 2.44 & 186.00 & 9.40 & 3.52 & 14 & \multirow{7}{*}{75} \\
\hline 2 & $\begin{array}{c}12 \\
\text { August } \\
2008\end{array}$ & $\begin{array}{l}71^{\circ} 30^{\prime} 749^{\prime \prime} \mathrm{N} \\
122^{\circ} 59^{\prime} 67^{\prime \prime} \mathrm{E}\end{array}$ & Olenyok & 16.4 & & & & & & & & & 22 & \\
\hline 3 & $\begin{array}{c}13 \\
\text { August } \\
2008\end{array}$ & $\begin{array}{l}71^{\circ} 78^{\prime} 533^{\prime \prime} \mathrm{N}, \\
123^{\circ} 73^{\prime} 743^{\prime \prime} \mathrm{E}\end{array}$ & Olenyok & 16.4 & 7.93 & 4.0 & 11.20 & 228.15 & 2.30 & 26.00 & 9.19 & 3.96 & 26 & \\
\hline 4 & $\begin{array}{c}13 \\
\text { August } \\
2008\end{array}$ & $\begin{array}{l}71^{\circ} 78^{\prime} 935^{\prime \prime} \mathrm{N}, \\
123^{\circ} 69^{\prime} 794^{\prime \prime} \mathrm{E}\end{array}$ & Olenyok & 17.4 & 6.78 & 4.0 & 6.40 & 67.46 & 1.34 & 33.00 & 9.81 & 2.64 & 40 & \\
\hline 5 & $\begin{array}{c}13 \\
\text { August } \\
2008\end{array}$ & $\begin{array}{l}71^{\circ} 81^{\prime} 098^{\prime \prime} \mathrm{N}, \\
123^{\circ} 68^{\prime} 292^{\prime \prime} \mathrm{E}\end{array}$ & Olenyok & 16.8 & 7.52 & 4.0 & 7.20 & 180.67 & 1.80 & 38.00 & 9.26 & 4.40 & 52 & \\
\hline 6 & $\begin{array}{c}13 \\
\text { August } \\
2008\end{array}$ & $\begin{array}{l}72^{\circ} 13^{\prime} 966^{\prime \prime} \mathrm{N}, \\
123^{\circ} 45^{\prime} 577^{\prime \prime} \mathrm{E}\end{array}$ & Olenyok & 17.1 & & & & & & & & & 21 & \\
\hline 7 & $\begin{array}{c}14 \\
\text { August } \\
2008\end{array}$ & $\begin{array}{l}72^{\circ} 55^{\prime} 621^{\prime \prime} \mathrm{N}, \\
122^{\circ} 04^{\prime} 922^{\prime \prime} \mathrm{E}\end{array}$ & Olenyok & 15.0 & 7.80 & 4.0 & 5.60 & 205.52 & 2.48 & 26.00 & 9.83 & 3.96 & 21 & \\
\hline 8 & $\begin{array}{l}\text { 17 July } \\
2009\end{array}$ & $\begin{array}{l}70^{\circ} 63^{\prime} 703^{\prime \prime} \mathrm{N}, \\
135^{\circ} 17^{\prime} 208^{\prime \prime} \mathrm{E}\end{array}$ & Yana & 14.6 & & & & & & & & & 6 & \multirow{5}{*}{40} \\
\hline 9 & $\begin{array}{l}18 \text { July } \\
2009\end{array}$ & $\begin{array}{l}70^{\circ} 75^{\prime} 302^{\prime \prime} \mathrm{N}^{\prime} \\
136^{\circ} 17^{\prime} 058^{\prime \prime} \mathrm{E}\end{array}$ & Yana & 14.5 & 7.13 & 0.8 & 14.80 & 101.00 & 1.90 & 40.00 & 10.33 & 2.64 & 15 & \\
\hline 10 & $\begin{array}{l}18 \text { July } \\
2009\end{array}$ & $\begin{array}{l}70^{\circ} 74^{\prime} 704^{\prime \prime} \mathrm{N}, \\
136^{\circ} 20^{\prime} 373^{\prime \prime} \mathrm{E}\end{array}$ & Yana & 15.1 & 6.69 & 0.9 & 4.80 & 45.96 & 1.10 & 20.00 & 10.60 & 2.64 & 0 & \\
\hline 11 & $\begin{array}{l}18 \text { July } \\
2009\end{array}$ & $\begin{array}{l}70^{\circ} 76^{\prime} 075^{\prime \prime} \mathrm{N}, \\
136^{\circ} 23^{\prime} 337^{\prime \prime} \mathrm{E}\end{array}$ & Yana & 14.2 & 7.13 & 0.9 & 8.40 & 92.06 & 1.60 & 50.00 & 10.28 & 2.64 & 23 & \\
\hline 12 & $\begin{array}{l}18 \text { July } \\
2009\end{array}$ & $\begin{array}{l}71^{\circ} 14^{\prime} 201^{\prime \prime} \mathrm{N}, \\
136^{\circ} 12^{\prime} 924^{\prime \prime} \mathrm{E}\end{array}$ & Yana & 14 & 7.13 & 0.9 & 14.00 & 104.26 & 1.90 & 50.00 & 10.30 & 2.64 & 31 & \\
\hline 13 & $\begin{array}{l}26 \text { June } \\
2009\end{array}$ & $\begin{array}{l}68^{\circ} 33^{\prime} 803^{\prime \prime} \mathrm{N}, \\
146^{\circ} 03^{\prime} 186^{\prime \prime} \mathrm{E}\end{array}$ & Indigirka & 14.2 & 7.77 & 0.2 & 79.20 & 97.65 & 2.40 & 69.00 & 9.86 & 3.52 & 0 & \multirow{13}{*}{7} \\
\hline 14 & $\begin{array}{l}\text { 26 June } \\
2009\end{array}$ & $\begin{array}{l}68^{\circ} 34^{\prime} 601^{\prime \prime} \mathrm{N}, \\
146^{\circ} 01^{\prime} 735^{\prime \prime} \mathrm{E}\end{array}$ & Indigirka & 14.9 & 7.24 & 0.1 & 8.00 & 52.21 & 2.00 & 33.00 & 9.45 & 5.72 & 5 & \\
\hline 15 & $\begin{array}{l}26 \text { June } \\
2009\end{array}$ & $\begin{array}{l}68^{\circ} 35^{\prime} 042^{\prime \prime} \mathrm{N}^{\prime} \\
145^{\circ} 99^{\prime} 79^{\prime \prime} \mathrm{E}\end{array}$ & Indigirka & 14.3 & 7.29 & 0.1 & 21.60 & 60.91 & 2.20 & 33.00 & 9.47 & 4.40 & 0 & \\
\hline 16 & $\begin{array}{l}27 \text { June } \\
2009\end{array}$ & $\begin{array}{l}68^{\circ} 56^{\prime} 854^{\prime \prime} \mathrm{N}^{\prime} \\
146^{\circ} 74^{\prime} 313^{\prime \prime} \mathrm{E}\end{array}$ & Indigirka & 14.2 & & & & & & & & & 0 & \\
\hline 17 & $\begin{array}{l}28 \text { June } \\
2009\end{array}$ & $\begin{array}{l}68^{\circ} 85^{\prime} 087^{\prime \prime} \mathrm{N}, \\
147^{\circ} 38^{\prime} 221^{\prime \prime} \mathrm{E}\end{array}$ & Indigirka & 14.7 & 7.43 & 0.1 & 10.00 & 90.12 & 1.90 & 33.00 & 9.61 & 3.52 & 0 & \\
\hline 18 & $\begin{array}{l}29 \text { June } \\
2009\end{array}$ & $\begin{array}{l}69^{\circ} 19^{\prime} 623^{\prime \prime} \mathrm{N} \\
147^{\circ} 49^{\prime} 045^{\prime \prime} \mathrm{E}\end{array}$ & Indigirka & 15.1 & & & & & & & & & 0 & \\
\hline 19 & $\begin{array}{l}\text { 29 June } \\
2009\end{array}$ & $\begin{array}{l}69^{\circ} 55^{\prime} 775^{\prime \prime} \mathrm{N}^{\prime \prime} \\
147^{\circ} 62^{\prime} 09^{\prime \prime} \mathrm{E}\end{array}$ & Indigirka & 15.5 & 7.55 & 0.1 & 44.80 & 90.65 & 0.50 & 33.00 & 9.00 & 4.40 & 0 & \\
\hline 20 & $\begin{array}{l}29 \text { June } \\
2009\end{array}$ & $\begin{array}{l}69^{\circ} 57^{\prime} 149^{\prime \prime} \mathrm{N}^{\prime} \\
147^{\circ} 62^{\prime} 26^{\prime \prime} \mathrm{E}\end{array}$ & Indigirka & 17.7 & 7.09 & 0.1 & 6.40 & 51.14 & 2.50 & 13.00 & 9.60 & 7.48 & 3 & \\
\hline 21 & $\begin{array}{l}29 \text { June } \\
2009\end{array}$ & $\begin{array}{l}69^{\circ} 58^{\prime} 76^{\prime \prime} \mathrm{N}, \\
147^{\circ} 59^{\prime} 92^{\prime \prime} \mathrm{E}\end{array}$ & Indigirka & 17.7 & 6.99 & 0.1 & 7.60 & 52.60 & 2.50 & 13.00 & 9.36 & 6.16 & 1 & \\
\hline 22 & $\begin{array}{l}30 \text { June } \\
2009\end{array}$ & $\begin{array}{l}70^{\circ} 52^{\prime} 957^{\prime \prime} \mathrm{N} \\
147^{\circ} 76^{\prime} 007^{\prime \prime} \mathrm{E}\end{array}$ & Indigirka & 15.5 & & & & & & & & & 0 & \\
\hline 23 & $\begin{array}{l}2 \text { July } \\
2009\end{array}$ & $\begin{array}{l}70^{\circ} 51^{\prime} 058^{\prime \prime} \mathrm{N} \\
147^{\circ} 68^{\prime} 349^{\prime \prime} \mathrm{E}\end{array}$ & Indigirka & 12.5 & 7.49 & 0.1 & 130.40 & 97.22 & 2.50 & 100.00 & 9.59 & 2.64 & 0 & \\
\hline 24 & $\begin{array}{l}2 \text { July } \\
2009\end{array}$ & $\begin{array}{l}70^{\circ} 53^{\prime} 011^{\prime \prime} \mathrm{N} \\
147^{\circ} 70^{\prime} 653^{\prime \prime} \mathrm{E}\end{array}$ & Indigirka & 14.1 & 7.47 & 0.1 & 88.40 & 80.66 & 2.40 & 168.00 & 9.42 & 3.08 & 0 & \\
\hline 25 & $\begin{array}{l}2 \text { July } \\
2009\end{array}$ & $\begin{array}{l}70^{\circ} 52^{\prime} 957^{\prime \prime} \mathrm{N}, \\
147^{\circ} 76^{\prime} 007^{\prime \prime} \mathrm{E}\end{array}$ & Indigirka & 14.1 & 7.44 & 0.1 & 84.80 & 80.71 & 2.50 & 168.00 & 9.36 & 3.08 & 0 & \\
\hline
\end{tabular}


Table 2. Cont.

\begin{tabular}{|c|c|c|c|c|c|c|c|c|c|c|c|c|c|c|}
\hline $\begin{array}{l}\text { Site } \\
\text { Num- } \\
\text { ber }\end{array}$ & $\begin{array}{l}\text { Sampling } \\
\text { Date }\end{array}$ & Coordinates & River & $\mathrm{T},{ }^{\circ} \mathrm{C}$ & $\begin{array}{l}\text { pH, } \\
\text { Epi }\end{array}$ & $\begin{array}{l}\text { Water Trans- } \\
\text { parency, } \\
\text { m }\end{array}$ & $\begin{array}{l}\text { Suspended } \\
\text { Matter, } \\
\text { mg/L }\end{array}$ & $\begin{array}{c}\Sigma \\
\text { Ions, } \\
\text { mg/L }\end{array}$ & $\underset{\mathrm{mg} / \mathrm{L}}{\mathrm{Si}}$ & $\begin{array}{l}P_{\text {total }} \\
\mu \mathrm{g} / \mathrm{L}\end{array}$ & $\underset{\mathrm{mg} / \mathrm{L}}{\mathrm{O}_{2}}$ & $\begin{array}{l}\mathrm{CO}_{2} \\
\mathrm{mg} / \mathrm{L}\end{array}$ & $\begin{array}{l}\text { Number } \\
\text { of } \\
\text { Species }\end{array}$ & $\begin{array}{c}\text { Total } \\
\text { Number } \\
\text { of Species } \\
\text { in the } \\
\text { River }\end{array}$ \\
\hline 26 & $\begin{array}{c}5 \\
\text { August } \\
2010\end{array}$ & $\begin{array}{l}68^{\circ} 31^{\prime} 505^{\prime \prime} \mathrm{N}^{\prime} \\
157^{\circ} 74^{\prime} 07^{\prime \prime} \mathrm{E}\end{array}$ & Kolyma & 16.7 & & & & & & & & & 16 & \\
\hline 27 & $\begin{array}{c}6 \\
\text { August } \\
2010\end{array}$ & $\begin{array}{l}68^{\circ} 71^{\prime} 893^{\prime \prime} \mathrm{N}^{\prime} \\
158^{\circ} 66^{\prime} 36^{\prime \prime} \mathrm{E}\end{array}$ & Kolyma & 15.9 & 7.60 & 1.9 & 5.20 & 126.06 & 1.34 & 80.00 & 9.84 & 5.28 & 16 & 31 \\
\hline 28 & $\begin{array}{c}6 \\
\text { August } \\
2010\end{array}$ & $\begin{array}{l}68^{\circ} 70^{\prime} 169^{\prime \prime} \mathrm{N} \\
158^{\circ} 70^{\prime} 129^{\prime \prime} \mathrm{E}\end{array}$ & Kolyma & 12.0 & 7.64 & 3.0 & 5.20 & 68.35 & 1.64 & 40.00 & 10.52 & 3.96 & 0 & \\
\hline 29 & $\begin{array}{c}6 \\
\text { August } \\
2010\end{array}$ & $\begin{array}{l}68^{\circ} 70^{\prime} 217^{\prime \prime} \mathrm{N}, \\
158^{\circ} 72^{\prime} 184^{\prime \prime} \mathrm{E}\end{array}$ & Kolyma & 14.4 & 7.48 & 1.9 & 5.20 & 92.00 & 2.04 & 380.00 & 10.63 & 3.52 & 0 & \\
\hline 30 & $\begin{array}{c}6 \\
\text { August } \\
2010\end{array}$ & $\begin{array}{l}68^{\circ} 57^{\prime} 6098^{\prime \prime} \mathrm{N} \\
159^{\circ} 60^{\prime} 2341^{\prime \prime} \mathrm{E}\end{array}$ & Kolyma & & & & & & & & & & 7 & \\
\hline 31 & $\begin{array}{c}7 \\
\text { August } \\
2010\end{array}$ & $\begin{array}{l}68^{\circ} 51^{\prime} 189^{\prime \prime} \mathrm{N}, \\
160^{\circ} 88^{\prime} 912^{\prime \prime} \mathrm{E}\end{array}$ & Kolyma & 13.9 & 7.63 & 1.4 & 5.60 & 124.01 & 1.34 & 120.00 & 10.30 & 5.28 & 9 & \\
\hline 32 & $\begin{array}{c}7 \\
\text { August } \\
2010\end{array}$ & $\begin{array}{l}68^{\circ} 46^{\prime} 522^{\prime \prime} \mathrm{N}, \\
160^{\circ} 80^{\prime} 042^{\prime \prime} \mathrm{E}\end{array}$ & Kolyma & 14.7 & 7.55 & 2.5 & 6.00 & 94.49 & 1.44 & 180.00 & 10.60 & 3.96 & 24 & \\
\hline 33 & $\begin{array}{c}7 \\
\text { August } \\
2010\end{array}$ & $\begin{array}{l}68^{\circ} 50^{\prime} 719^{\prime \prime} \mathrm{N}, \\
160^{\circ} 97^{\prime} 508^{\prime \prime} \mathrm{E}\end{array}$ & Kolyma & 14.4 & 7.26 & 1.4 & 5.60 & 90.32 & 1.44 & 180.00 & 9.89 & 3.96 & 0 & \\
\hline
\end{tabular}

In the Yana River, 40 species of the genera Mallomonas $(n=11)$, Synura $(n=19)$, Chrysosphaerella $(n=3)$, Paraphysomonas $(n=3)$, and Spiniferomonas $(n=4)$ were identified (Table 1). Hydrochemical parameters were relatively stable across the sampling stations of this river. Only water temperature dropped from $14.6^{\circ} \mathrm{C}$ at the southernmost station 8 to $14{ }^{\circ} \mathrm{C}$ at the most downstream sampling station 12 . In contrast, the species diversity of silica-scaled chrysophytes increased from the south (6 species) to north (31 species). This trend may be attributed to the progression of vegetation peak, which typically occurs at high latitudes between mid- and late summer. Paraphysomonas cf. punctata ssp. simplicior, Spiniferomonas cornuta, Mallomonas actinoloma, Synura praefracta, Synura nygaardii, and Synura leptorhabda showed scattered distribution (including rare species). Moreover, Mallomonas parvula, Synura leptorhabda, and Synura sp. 1 were detected only in Yana.

The species composition in the Indigirka River was the least diverse, with only seven species of Mallomonas (2) and Synura (5). Interestingly, no species of the genus Paraphysomonas and Lepidochromonas spp. were found in the river (Table 1). The widest ranges of some hydrochemical parameters were recorded in this habitat (Table 2). In this river, water temperature and $\mathrm{pH}$ values are favourable for the growth of chrysophytes. However, low transparency and high concentrations of suspended solids limit the development of phytoplankton, including silica-scaled chrysophytes, at the majority of the stations. High turbidity might be a greater problem for non-photosynthetic species, such as Paraphysomonas spp. In the present study, silica-scaled chrysophytes were detected at only 3 of the 13 stations. All species detected in this river, except Synura praefracta, are cosmopolitan and ubiquitous.

In the Kolyma River, 31 species belonging to the genera Chrysosphaerella $(n=2)$, Paraphysomonas $(n=2)$, Spiniferomonas $(n=2)$, Mallomonas $(n=9)$, and Synura $(n=16)$ (Table 1) were detected. Of these, Mallomonas caudata, Synura spinosa, and Synura spinosa f. longispina were the most frequently detected species at the study sites. Similarly, to the trends in Indigirka, the same hydrochemical parameters showed wide ranges in Kolyma. For instance, water temperature varied between $12{ }^{\circ} \mathrm{C}$ and $16.7^{\circ} \mathrm{C}$, and no chrysophyceans were found in the regions with the lowest temperatures $\left(12-14.4^{\circ} \mathrm{C}\right)$. Similarly, total phosphorus content also showed a wide range ( 40 to $\left.380 \mu \mathrm{g} \cdot \mathrm{L}^{-1}\right)$, and no chrysophyceans were found in regions with the lowest or highest concentration. The distribution of silica-scaled chrysophytes from the southernmost sampling site to the northernmost one was uneven. Specifically, 16 species were identified at the southernmost sampling site, 
which disappeared from stations 28 and 29 with a drop in temperature and a rise in total phosphorus content. Farther northward, where the hydrochemical parameters remained stable, the species richness of silica-scaled chrysophytes increased, reaching the maximum for this river (24 species at station 32). Only three species, namely Paraphysomonas vulgaris, Synura nygaardii, and Synura praefracta, showed scattered distribution, and the rest were cosmopolitan and ubiquitous.

Paraphysomonas uniformis ssp. hemiradia, Mallomonas acaroides, Mallomonas alpina, Mallomonas crassisquama, Mallomonas tonsurata, Synura borealis, Synura petersenii, Synura praefracta, Synura cf. americana, and Synura conopea were the most frequently detected species. All these species, except Synura praefracta, are cosmopolitan and ubiquitous. The prevalent taxa in estuaries can be divided into two groups based on their response to $\mathrm{pH}$ gradient [3]: $\mathrm{pH}$-indifferent species, such as Mallomonas crassisquama and Synura petersenii, which occur in waters with a $\mathrm{pH}$ of $\sim 7$, and alkaliphilic species, such as Mallomonas acaroides, Mallomonas alpina, Mallomonas crassisquama, and Mallomonas tonsurata, which prefer waters with a high $\mathrm{pH}(>7)$.

\subsection{Addition of Autecology of Rare Species}

Notably, some rare species were observed in the estuaries of the studied rivers, and majority of them were detected at station 5 in Olenyok. The eight species described for the first time in the waters of Russia were found only at this station. We classified the species as rare if they had been described a long time ago in few studies, or if they had been described only recently because of their poorly known autecology, as in the case of Synura cf. vinlandica, Synura cf. cornuta, and Synura cf. soroconopea.

Synura cf. vinlandica (Figure S6o-q) was recently described based on molecular and morphological studies of isolated colonies in samples collected from Newfoundland, Canada [64]. We classified our samples as Synura cf. vinlandica based on the morphological features of scales. In the present study, we detected this species at station 7 in Olenyok ( $\left.\mathrm{pH}=7.80, \mathrm{~T}=15.0^{\circ} \mathrm{C}\right)$ (Table 2$)$.

Synura cf. cornuta (Figure S6m,n) was recently described based on molecular and morphological studies of isolated colonies in samples collected from Newfoundland, Canada [64]. We classified our samples as Synura cf. cornuta based on the most distinct morphological feature of scales possessing a 'narrow and prominent tip, resembling a horn' [64]. In the present study, we detected this species at stations 1 and 3 in Olenyok River ( $\left.\mathrm{pH}=7.89-7.93, \mathrm{~T}=16-16.4^{\circ} \mathrm{C}\right)$ (Table 2).

Synura cf. soroconopea (Figure S7a,b) Jo, Shin, Kim \& Siver was described based on molecular and morphological studies of isolated colonies in samples collected from small ponds in South Korea [65]. In the present study, we frequently detected this species at different stations in Olenyok, Yana, and Kolyma ( $\left.\mathrm{pH}=6.69-7.93, \mathrm{~T}=14-17.4{ }^{\circ} \mathrm{C}\right)$ (Table 2).

Chrysosphaerella rotundata (Figure S1f) was described from a water body in Finland [66]. Before its description, chrysophytes with rounded scales similar to Chrysosphaerella rotundata were found in Germany (classified by the author as Chrysosphaerella brevispina in [67]: Figure 76). The species has also been recorded in the Selenga Delta of Russia [13]. It has been reported to inhabit waters with temperatures between $5^{\circ} \mathrm{C}$ [66] and $19{ }^{\circ} \mathrm{C} \mathrm{[67],} \mathrm{pH}$ between 7 [66] and 7.85 [13], and conductivity between 40 [67] and $340 \mu \mathrm{S} \cdot \mathrm{cm}^{-1}$ [67]. In the present study, this species was detected at station 5 in Olenyok $\left(\mathrm{pH}=7.52, \mathrm{~T}=16.8{ }^{\circ} \mathrm{C}\right)$ (Table 2).

Paraphysomonas circumvallata (Figure S1q) was described from a water body in Danish [68]. It has also been recorded in Greece [69], North America [70,71], Germany [72], New Zealand [73], and the Pojo Bay of Finland [74]. It was recorded for the last time in the Gulf of Finland, Baltic Sea [75]. However, only one previous study has reported the ideal hydrochemical parameters for the growth of this species in a lake in Germany $(\mathrm{pH}=7.8$, conductivity $136 \mu \mathrm{S} \cdot \mathrm{cm}^{-1}$ ) [72]. In the present study, this species was detected at stations 3 , 4 , and 5 in Olenyok $\left(\mathrm{pH}=6.78-7.93, \mathrm{~T}=16.4-17.4^{\circ} \mathrm{C}\right)($ Table 2$)$. 
Lepidochromonas eiffelii (Figure S2f,g) was described from a water body in Danish [68]. It has also been recorded in England [76], Chile [77], North America [78], Germany [72], Hungary [79], and Austria [80], China [81]. It has been reported to inhabit waters with temperatures between $0{ }^{\circ} \mathrm{C}$ [68] and $20^{\circ} \mathrm{C}$ [81], pH between 6.2 [72] and 8.7 [81], and conductivity between 102 [80] and $490 \mu \mathrm{S} \cdot \mathrm{cm}^{-1}$ [72]. In the present study, this species was detected at station 5 in Olenyok $\left(\mathrm{pH}=7.52, \mathrm{~T}=16.8^{\circ} \mathrm{C}\right)($ Table 2$)$.

Lepidochromonaselegantissima (Figure $\mathrm{S} 2 \mathrm{~h}$ ) was described from a water body in North America [70]. It has also been recorded in North America [71], Denmark [82], and Baltic Sea ice stations in Finland [83]. It has been reported to inhabit waters with temperatures between $0{ }^{\circ} \mathrm{C}$ [83] and $3{ }^{\circ} \mathrm{C}$ [82], $\mathrm{pH}$ between 5.6 [82] and 7.7 [83], and conductivity of $70 \mu \mathrm{S} \cdot \mathrm{cm}^{-1}$ [82]. In the present study, this species was detected at station 5 in Olenyok $\left(\mathrm{pH}=7.52, \mathrm{~T}=16.8^{\circ} \mathrm{C}\right)$ (Table 2).

Lepidochromonas quadrispina (Figure S2e) was described from a water body in Danish [68]. It has also been recorded in Greece [69], Finland [11,84,85], and the Vorkuta tundra of Russia [22]. It has been reported to inhabit waters with temperatures between $10.7^{\circ} \mathrm{C}$ and $13^{\circ} \mathrm{C}$ [22], pH between 6.7 and 6.8 [84], and conductivity between 23 [22] and $47 \mu \mathrm{S} \cdot \mathrm{cm}^{-1}$ [84]. In the present study, this species was detected at station 5 in Olenyok $\left(\mathrm{pH}=7.52, \mathrm{~T}=16.8^{\circ} \mathrm{C}\right)($ Table 2$)$.

Lepidochromonasundulata (Figure S2k) was described from a water body in England [76]. It has also been recorded in Finland [85], Hungary [79], and the Vorkuta tundra of Russia [22]. It has been reported to inhabit waters with temperatures between $10.5^{\circ} \mathrm{C}$ ([79]) and $17{ }^{\circ} \mathrm{C}$ [22], pH between 6.2 and 6.8 [79], and conductivity between 24 [22] and $240 \mu \mathrm{S} \cdot \mathrm{cm}^{-1}$ [79]. In the present study, this species was detected at station 5 in Olenyok $\left(\mathrm{pH}=7.52, \mathrm{~T}=16.8^{\circ} \mathrm{C}\right)($ Table 2$)$.

Spiniferomonas conica (Figure S3j) was described from a water body in Japan [86]. It has also been recorded in North America [87], Greenland [88], and the Lake Labynkyr in Russia [48]. It has been reported to inhabit waters with temperatures between $10^{\circ} \mathrm{C}$ [88] and $15.8^{\circ} \mathrm{C}$ [48], pH between 5.9 [88] and 7.63 [48], and conductivity between 11 [88] and $38 \mu \mathrm{S} \cdot \mathrm{cm}^{-1}$ [48]. In the present study, this species was detected at station 2 in Olenyok $\left(\mathrm{T}=16.4^{\circ} \mathrm{C}\right)($ Table 2$)$.

Spiniferomonas minuta (Figure S3k) was described from a water body in North America $[89,90]$. It has also been recorded in North America (as Spiniferomonas takahashii in Nicholls, [87]: Figure 38; [89]), Finland [84], the Gulf of Bothnia, Baltic Sea [91], and small lakes of the Lower Yenisei Basin in Russia [27]. It has been reported to inhabit waters with temperatures between $4.5^{\circ} \mathrm{C}$ [27] and $13.1^{\circ} \mathrm{C}$ [84], pH between 5.6 [91], and 8.4 [27], conductivity between 20 [90] and $150 \mu \mathrm{S} \cdot \mathrm{cm}^{-1}$ [27]. In the present study, this species was detected at station 4 in Olenyok $\left(\mathrm{pH}=6.78, \mathrm{~T}=17.4^{\circ} \mathrm{C}\right)$ (Table 2).

Spiniferomonas takahashii (Figure S31) was described from a water body in North America [87]. It has also been recorded in North America [90,92], the Gulf of Bothnia, Baltic Sea [91], and the Lake Baikal in Russia [93]. It has been reported to inhabit waters with temperatures between $8{ }^{\circ} \mathrm{C}$ and $20^{\circ} \mathrm{C}$ [90], $\mathrm{pH}$ between 5.8 [91] and 8 [93], and conductivity between 34 [90] and $110 \mu \mathrm{S} \cdot \mathrm{cm}^{-1}$ [93]. In the present study, this species was detected at station 5 in Olenyok $\left(\mathrm{pH}=7.52, \mathrm{~T}=16.8^{\circ} \mathrm{C}\right)$ (Table 2).

Synura petersenii f. taymyrensis (Figure S7f) was described from a water body in the Taymyr Peninsula of Russia [9]. In the present study, Synura petersenii f. taymyrensis was detected at station 4 in Olenyok $\left(\mathrm{pH}=6.78, \mathrm{~T}=17.4^{\circ} \mathrm{C}\right)$ (Table 2), representing the first record of this species since its description.

\subsection{Undetermined Species}

Eleven species showing scale morphology different from all known species were found in areas with high diversity of silica-scaled chrysophytes. These are likely to be new species, and their scales and spines are described below.

We found Chrysosphaerella sp. (Figure S1c,d) bearing two types of scales, including round (2.3-2.9 $\mu \mathrm{m}$ diameter) or oval (2.4-2.6 $\mu \mathrm{m}$ long and 1.4-1.7 $\mu \mathrm{m}$ wide) ones. Both 
types of scales were ornamented with series of elongated, round ridges, forming a serrated pattern. The considerably large, round ridges differentiate these scales from structures in other known species. In some oval scales, a secondary pattern was observed under the ridges (Figure S1d). In the present study, this species was detected at stations 4 and 5 in Olenyok ( $\left.\mathrm{pH}=6.78-7.52, \mathrm{~T}=16.8-17.4^{\circ} \mathrm{C}\right)$ (Table 2$)$.

We found Paraphysomonas sp. 1 (Figure S1h) bearing a round to oval, rimless baseplate (diameter, 0.9-1.1 $\mu \mathrm{m}$ ). The plate bore 2.4-2.8- $\mu \mathrm{m}$-long spines, tapering in a pinched tip. The proximal part of the spine $(0.5 \mu \mathrm{m}$ from the baseplate) was darker in colour, suggesting that this part was denser than the rest of the spine. In the present study, this species was detected at stations 4, 5, and 6 in Olenyok ( $\mathrm{pH}=6.78-7.51, \mathrm{~T}=16.8-17.4^{\circ} \mathrm{C}$ ) (Table 2).

We found Paraphysomonas sp. 2 (Figure S1k) bearing a round baseplate (diameter, $0.6 \mu \mathrm{m}$ ) with a highly incurved border (width $0.04 \mu \mathrm{m}$ ). The margins of the baseplate possessed small perforations. A spine (length $0.9 \mu \mathrm{m}$ ) protruded from the centre of the round baseplate, and it was rounded at the tip. In the present study, this species was detected at station 5 in Olenyok $\left(\mathrm{pH}=7.52, \mathrm{~T}=16.8^{\circ} \mathrm{C}\right)$ (Table 2).

We found Paraphysomonas sp. 3 (Figure S2a-c) bearing elliptical, deeply undulate plate-scales $(0.9-1.5 \times 1.3-1.9 \mu \mathrm{m})$ with a deep crater-like depression (crater-scales). The rim was $0.2-0.5 \mu \mathrm{m}$ thick, with a ring of 70-74 small pegs along its inner edge on the distal side. Five to seven large apertures (diameter 0.08-0.2 $\mu \mathrm{m}$ ) were located in the crater-like depression. In the present study, this species was detected at stations 4 and 5 in Olenyok $\left(\mathrm{pH}=6.78-7.52, \mathrm{~T}=16.8-17.4^{\circ} \mathrm{C}\right)$ (Table 2).

We found Paraphysomonas sp. 4 (Figure S2d) bearing an elliptical baseplate $(1.4-1.5 \times 1.6-1.8 \mu \mathrm{m})$. The inner edge of the baseplate was ornamented with five rows of 62-85 min marginal pegs on the distal side. In the present study, this species was detected at station 5 in Olenyok $\left(\mathrm{pH}=7.52, \mathrm{~T}=16.8^{\circ} \mathrm{C}\right)$ (Table 2).

We found Lepidochromonas sp. 1 (Figure S2l) bearing perforated, elliptical plate-scales $(1 \times 1.2 \mu \mathrm{m})$. The plate-scale perforations varied in size and were randomly distributed. In the central part of the scale, there were four large apertures (diameter, $0.2 \mu \mathrm{m}$ ). In the present study, this species was detected at station 5 in Olenyok $\left(\mathrm{pH}=7.52, \mathrm{~T}=16.8{ }^{\circ} \mathrm{C}\right)$ (Table 2).

We found Lepidochromonas sp. 2 (Figure S2n) bearing crown-like scales with an elliptical base, which was wider on one side of the scale $(0.6 \mu \mathrm{m})$ but slightly narrower on the other $(0.5 \mu \mathrm{m})$. The base of the crown-like scales possessed six long, perpendicular shafts, distally connected with the arches. The arch structures were perforated. In the present study, this species was detected at station 5 in Olenyok $\left(\mathrm{pH}=7.52, \mathrm{~T}=16.8^{\circ} \mathrm{C}\right)$ (Table 2).

We found Synura sp. 1 (Figure S5o) bearing oval body scales $(2.8 \times 2.3 \mu \mathrm{m})$ with an obtuse (flat) tip. Spine was unknown. There was no upturned edge around the periphery of the scale. The basal plate showed regular pore ornamentation. The vermiform ornamentation area on the distal portion was absent. In the distal flange area, 15 ribs $(0.27 \mu \mathrm{m}$ long) were present, bearing large pores between them. In the present study, this species was detected at station 12 in Yana River $\left(\mathrm{pH}=7.13, \mathrm{~T}=14{ }^{\circ} \mathrm{C}\right)$ (Table 2).

We found Synura sp. 2 (Figure S7c,d) bearing body scales (3.8-4.3 $\mu \mathrm{m}$ long and 2.3-2.4 $\mu \mathrm{m}$ wide) possessing a basal plate with a centrally raised keel protruding into an acute tip. The keel was anteriorly widened and had ornamented pores (diameter, 80-95 nm). Numerous small pores ornamented the basal plate. Numerous struts (34-36) regularly extended from the keel toward the scale perimeter. Short struts were present between the primary ones. One to two short struts spurred off from one side of the keel, and a short strut was present on the other side at the scale perimeter. A secondary layer running along the scale perimeter was present between the struts in the upper part of the scale. There were randomly arranged pores (diameter, 80-95 nm) close to the secondary layer. In the present study, this species was detected at stations 4, 6, and 7 Olenyok, at station 9 in Yana, and at stations 27 and 32 in Kolyma $\left(\mathrm{pH}=6.78-7.80, \mathrm{~T}=14.5-17.4^{\circ} \mathrm{C}\right)$ (Table 2).

We found Synura sp. 3 (Figure S6i) possessing body scales $(4.3 \mu \mathrm{m}$ long and $1.8 \mu \mathrm{m}$ wide) bearing a basal plate with a centrally raised keel protruding into an acute tip. The 
keel, ornamented with small pores, widened anteriorly, then tapered and widened again. Transverse folds interconnected numerous struts (38). In the present study, this species was detected at station 4 in Olenyok $\left(\mathrm{pH}=6.78, \mathrm{~T}=17.4^{\circ} \mathrm{C}\right)$ (Table 2).

We found Synura sp. 4 (Figure S7i-1) possessing elongated body scales (3.0-3.8 $\mu \mathrm{m}$ long and 1.1-1.5 $\mu \mathrm{m}$ wide). The keel of the body scales was much reduced and ornamented with small pores (diameter, 34-60 nm). Struts (26-38), interconnected with numerous transverse ribs, regularly extended outward from the keel toward the scale perimeter. Short struts were present between the primary ones. One to two short struts spurred off from one side of the keel, and a short strut was present on the other side at the scale perimeter. In the present study, this species was detected at stations 3, 4, 5, and 7 in Olenyok and at stations 8,11 , and 12 of Yana $\left(\mathrm{pH}=6.78-7.93, \mathrm{~T}=14.0-17.4^{\circ} \mathrm{C}\right)($ Table 2$)$.

\subsection{Physicochemical Parameters and Species Composition and Richness}

Samples were collected during summer when water temperature of the northern rivers is relatively high $\left(12.0-17.7^{\circ} \mathrm{C}\right)$ (Table 2$)$. Chrysophyceans were not found at the minimum recorded temperature (station $28: 12.0^{\circ} \mathrm{C}$ ). The maximum number of species was recorded in Kolyma at temperatures of $14.7^{\circ} \mathrm{C}$ (station 32, $n=24$ ), $15.9^{\circ} \mathrm{C}$ (station 27, $n=16$ ), and $16.7^{\circ} \mathrm{C}$ (station $\left.26, n=16\right)$. Similarly, few species were found $(n=3$ at station 20 and $n=1$ at station 21 in Indigirka) at the maximum recorded temperature $\left(17.7^{\circ} \mathrm{C}\right)$. Amongst the studied rivers, the highest number of species $(n=52)$ was found in Olenyok at the temperature of $16.8^{\circ} \mathrm{C}$, followed by Yana $(n=31)$ at the temperature of $14.0^{\circ} \mathrm{C}$, Kolyma $(n=24)$ at the temperature of $14.7^{\circ} \mathrm{C}$, and Indigirka $(n=5)$ at the temperature of $14.9^{\circ} \mathrm{C}$. Therefore, the temperature regime of $14.0-16.8^{\circ} \mathrm{C}$ may be considered optimal for silica-scaled chrysophytes, under otherwise equal parameters.

During the present study, we recorded neutral to slightly alkalescent $\mathrm{pH}$ values in all rivers except Yana. The highest species richness of chrysophyceans was recorded at closely situated stations 4 and 5 in Olenyok at both neutral and slightly acidic $\mathrm{pH}(6.78$, the lowest value at which species were found) or slightly alkaline (7.52) values. Of note, the species composition of silica-scaled chrysophytes at these two stations was different (Table 2).

Low water transparency is typical of Indigirka and Yana, because easily erodible sandy clay and Upper Paleozoic and Mesozoic deposits [94] dominate the drainage basins at the sampling sites in both rivers. Permafrost outcrops and intense thawing of permafrost in summer enhance the processes of bank erosion downstream the rivers, thus increasing the concentration of suspended solids and decreasing water transparency. The highest concentration of suspended solids was recorded in Indigirka $\left(130.40 \mathrm{mg} \cdot \mathrm{L}^{-1}\right)$, and no silica-scaled chrysophytes were found even at turbidity of $21.60 \mathrm{mg} \cdot \mathrm{L}^{-1}$ (Table 2). Water transparency of Kolyma was higher, being comparable to that at some stations in Olenyok. The highest water transparency was recorded in the most species rich location, Olenyok River.

In general, the studied rivers have low salt content, with the exception of Olenyok, which shows moderate salinity. The highest species richness was recorded at salt concentrations ranging from 67.46 (station $4, n=40$ ) to $228.15 \mathrm{mg} \cdot \mathrm{L}^{-1}$ (station $3, n=26$ ). This range of salt concentration does not limit the development of silica-scaled chrysophytes.

Silicon concentration showed a wide range $\left(0.5\right.$ to $\left.2.5 \mathrm{mg} \cdot \mathrm{L}^{-1}\right)$. Algal blooms are known to depend on silicon concentration in water. The lowest silicon concentration $\left(1.34 \mathrm{mg} \cdot \mathrm{L}^{-1}\right)$ at which chrysophycean species were found was recorded in Kolyma (station 27), whilst the highest concentration $\left(2.48 \mathrm{mg} \cdot \mathrm{L}^{-1}\right)$ was recorded in Olenyok (station 7). Almost all stations in Indigirka showed comparable silicon concentrations $\left(1.9-2.5 \mathrm{mg} \cdot \mathrm{L}^{-1}\right)$, except station $19\left(0.5 \mathrm{mg} \cdot \mathrm{L}^{-1}\right)$; however, very low numbers of silica-scaled chrysophytes were recorded in this river (stations 20,n=3 and station 21, $n=1$ ), and none station 19 with the minimum concentration of silicon $\left(0.5 \mathrm{mg} \cdot \mathrm{L}^{-1}\right)$.

Some samples from Indigirka, Olenyok, and Kolyma showed remarkably high total phosphorus content $\left(168,186\right.$, and $380 \mu \mathrm{g} \cdot \mathrm{L}^{-1}$, respectively). The total phosphorus content of the majority of the remaining samples was low and did not exceed $100 \mu \mathrm{g} \cdot \mathrm{L}^{-1}$. Despite 
the higher phosphorus content at some sites, the biological availability of this element was apparently low. In the present study, chrysophyceans were found at total phosphorus concentrations of between $13 \mu \mathrm{g} \cdot \mathrm{L}^{-1}$ in Indigirka (stations 20,n=3 and station 21, n=1) and $80 \mu \mathrm{g} \cdot \mathrm{L}^{-1}$ in Kolyma (station $\left.27, n=15\right)$. The maximum number of species (23-52) was recorded at total phosphorus concentrations within $26-180 \mu \mathrm{g} \cdot \mathrm{L}^{-1}$, which may be optimal for the development of silica-scaled chrysophytes in the study area. The highest total phosphorus concentration at which chrysophyceans were found was recorded in Kolyma $\left(180 \mu \mathrm{g} \cdot \mathrm{L}^{-1}\right.$; station $\left.32, n=24\right)$ and Olenyok $\left(186 \mu \mathrm{g} \cdot \mathrm{L}^{-1}\right.$; station $\left.1, n=14\right)$ (Table 2$)$.

There were no considerable variations in the concentration of dissolved oxygen (9.19-10.63 $\left.\mathrm{mg} \cdot \mathrm{L}^{-1}\right)$ amongst the sampling stations, indicating a favourable oxygen regimen and good aeration of waters. The concentration of dissolved oxygen within the above limits did not affect the total number of species in the sample. Meanwhile, the concentration of carbon dioxide was low (2.64-7.48 $\left.\mathrm{mg} \cdot \mathrm{L}^{-1}\right)$ (Table 2).

Overall, the maximum number of species $(n=52)$ was recorded at station 5 in Olenyok, where the values of all physical and chemical parameters of the river environment were average (Table 2).

Furthermore, we statistically evaluated the effects of the studied physical and chemical factors of the river environment on species richness and composition of silica-scaled chrysophytes. PERMANOVA (Table 3) revealed that factors including the provenance of a sample from a certain river; distance of the sampling site from the estuary (species composition changed toward the sea); water temperature, transparency, and colour; and concentration of carbon dioxide $\left(\mathrm{CO}_{2}\right)$, magnesium ions $\left(\mathrm{Mg}^{2+}\right)$, total iron $\left(\mathrm{Fe}_{\text {total }}\right)$, surfactants, and oil products, truly affected the species composition of chrysophycean algae in all rivers. Regarding the coefficient of variation $\left(\mathrm{R}^{2}\right)$, the most important factor determining the species composition in the samples was the provenance of a sample from a certain site of the river $\left(R^{2}=0.29\right)$. The importance of other factors, even though they reliably affected the species composition in samples, was low $\left(R^{2}=0.1\right)$.

In NMDS scatter plot (see biplot in Figure 2), the sampling sites in Olenyok formed a close cluster in the right part of the reference frame. This cluster also included three sampling sites in Yana. The species composition of chrysophyceans was similar at all sites in the cluster, but different from that at the remaining sites. The remaining sampling sites were scattered in the left and upper parts of the reference frame. These sites differed in terms of species composition, both from one another and from sampling sites in Olenyok and Yana that formed the above-mentioned cluster.

Three groups of environmental parameters reliably affecting the species composition in samples could be identified based on the direction of gradient vectors (see biplot in Figure 2). The first group included codirectional vectors of water temperature, water transparency, and magnesium ion $\left(\mathrm{Mg}^{2+}\right)$ concentration, which were positively correlated with one another. The second group included surfactant concentration and distance of a sampling site from the estuary, which were positively correlated with each other. The third group included codirectional vectors of carbon dioxide $\left(\mathrm{CO}_{2}\right)$, oil product, and total iron $\left(\mathrm{Fe}_{\text {total }}\right)$ concentration and water colour, which were positively correlated with one another. The vectors of the first and second group were contradirectional and constituted an assembly of negatively correlated parameters. Water temperature, water transparency, and magnesium ion $\left(\mathrm{Mg}^{2+}\right)$ concentration increased but surfactant concentration decreased as the sampling sites approached the river estuary. Meanwhile, the environmental parameters in the first and second groups (water temperature and transparency, magnesium ion $\left(\mathrm{Mg}^{2+}\right.$ ) and surfactant concentration, and distance of a sampling site from the estuary) constituted an assembly of factors that complementarily affected the species composition in samples. Parameters in the third group (carbon dioxide $\left(\mathrm{CO}_{2}\right)$, oil product, and total iron $\left(\mathrm{Fe}_{\text {total }}\right)$ concentration and water colour) exerted another type of effect, which was not related to the mechanisms of effect of parameters in the first and second groups. 
Table 3. PERMANOVA analysis results on reliability of impact of abiotic environmental parameters on species richness in samples. The reliable impact of a factor is seen at $p$ value $\leq 0.05$. All reliably affecting factors are in bold.

\begin{tabular}{|c|c|c|}
\hline Factor Names & $\mathbf{R}^{2}$ Covariance Coefficient & $p$ Value \\
\hline Sampling river & 0.290 & 0.001 \\
\hline Distance to the mouth, $\mathrm{km}$ & 0.098 & 0.049 \\
\hline $\mathrm{T},{ }^{\circ} \mathrm{C}$ & 0.090 & 0.042 \\
\hline Water transparency, m & 0.097 & 0.031 \\
\hline $\mathrm{pH}, \mathrm{Epi}$ & 0.065 & 0.275 \\
\hline Suspended matter, mg/L & 0.062 & 0.373 \\
\hline Color, Pt-Co & 0.105 & 0.019 \\
\hline $\mathrm{O}_{2}, \mathrm{mg} / \mathrm{L}$ & 0.068 & 0.228 \\
\hline Oxygen saturation, $\%$ & 0.068 & 0.232 \\
\hline $\mathrm{CO}_{2}, \mathrm{mg} / \mathrm{L}$ & 0.097 & 0.048 \\
\hline$\Sigma_{\text {ions }}, \mathrm{mg} / \mathrm{L}$ & 0.065 & 0.300 \\
\hline Water hardness, mg-equ/L & 0.069 & 0.249 \\
\hline $\mathrm{Ca}^{2+}, \mathrm{mg} / \mathrm{L}$ & 0.056 & 0.505 \\
\hline $\mathrm{Mg}^{2+}, \mathrm{mg} / \mathrm{L}$ & 0.099 & 0.025 \\
\hline $\mathrm{Na}^{+}, \mathrm{mg} / \mathrm{L}$ & 0.052 & 0.564 \\
\hline $\mathrm{K}^{+}, \mathrm{mg} / \mathrm{L}$ & 0.060 & 0.415 \\
\hline $\mathrm{HCO}_{3}{ }^{-}, \mathrm{mg} / \mathrm{L}$ & 0.063 & 0.342 \\
\hline $\mathrm{Cl}^{-}, \mathrm{mg} / \mathrm{L}$ & 0.045 & 0.727 \\
\hline $\mathrm{SO}_{4}{ }^{2-}, \mathrm{mg} / \mathrm{L}$ & 0.062 & 0.364 \\
\hline $\mathrm{NH}_{4}, \mathrm{mg} / \mathrm{L}$ & 0.075 & 0.204 \\
\hline $\mathrm{NO}_{2}, \mathrm{mg} / \mathrm{L}$ & 0.062 & 0.199 \\
\hline $\mathrm{NO}_{3}, \mathrm{mg} / \mathrm{L}$ & 0.077 & 0.138 \\
\hline $\mathrm{PO}_{4}, \mathrm{mg} / \mathrm{L}$ & 0.071 & 0.205 \\
\hline $\mathrm{P}_{\text {total }}, \mu \mathrm{g} / \mathrm{L}$ & 0.055 & 0.515 \\
\hline $\mathrm{Si}, \mathrm{mg} / \mathrm{L}$ & 0.059 & 0.419 \\
\hline $\mathrm{COD}, \mathrm{mg} / \mathrm{L}$ & 0.074 & 0.177 \\
\hline BOD5, mg/L & 0.063 & 0.328 \\
\hline $\mathrm{Fe}_{\text {total }}, \mathrm{mg} / \mathrm{L}$ & 0.102 & 0.025 \\
\hline Phenols, mg/L & 0.066 & 0.298 \\
\hline Surfactants, mg/L & 0.133 & 0.007 \\
\hline Petrochemicals, mg/L & 0.121 & 0.009 \\
\hline
\end{tabular}

The species richness in samples increased with increasing water temperature, water transparency, and magnesium ion $\left(\mathrm{Mg}^{2+}\right)$ concentration, as reflected by the direction of gradient vector on the NMDS scatter plot (Figure 2). Similarly, the species richness in samples increased as the sampling site approached the estuary (i.e., the sea) but decreased as surfactant concentration increased. The other physical and chemical parameters, within the limits of their variation range, did not significantly affect species composition in the samples. 


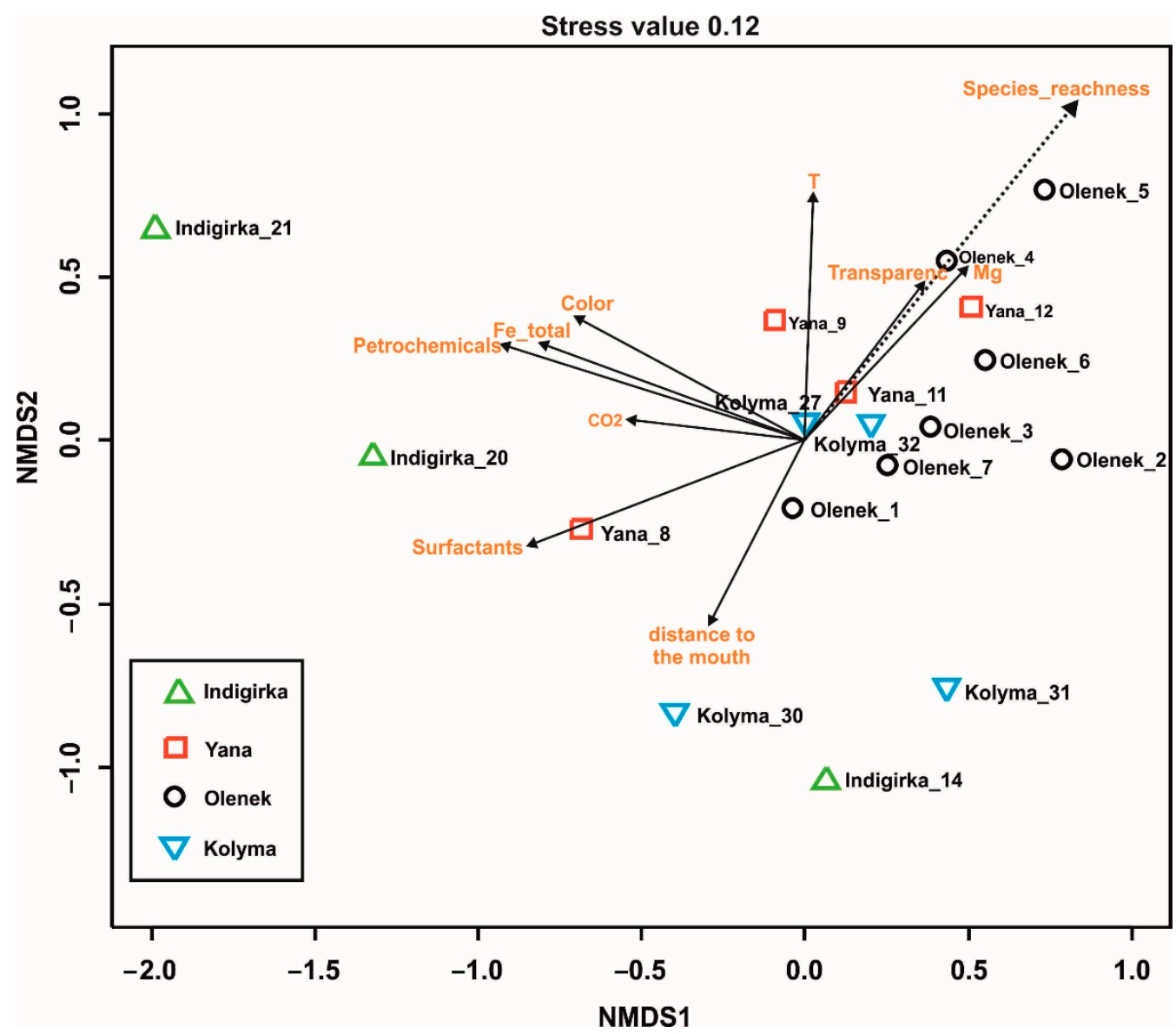

Figure 2. Scatter plot of sampling sites done on results of multidimensional scaling based on the Jaccard distances showing difference by species lists. Arrows designate gradients of quantitative factors reliably affecting the species composition in samples (Table 3). Broken lines show the species richness gradient vector in samples.

\subsection{Specific Morphology of Some Chrysophytes Found in the Study Area}

The present investigation of the species richness of silica-scaled chrysophytes revealed some peculiarities in the structure of scales in Spiniferomonas bourrellyi, Synura mammillosa, and Synura echinulate.

Synura echinulate is a cosmopolitan species that mainly occurs in oligotrophic and slightly acidic waters. In Synura echinulate collected in the present study, the scales occasionally lacked the labyrinth pattern (Figure S5i, station 12) on the distal third and instead presented a dotted pattern, which was only slightly similar to the former pattern (Figure S5j, station 5). In some other samples, the scales completely lacked the papillar ornament (Figure S5k, station 32) and possessed a weakly pronounced, upturned rim surrounding one-half to two-thirds of the scale (Figure S5j,k). Given the sufficient concentration of silicon in the waters of these three stations $\left(1.44-1.90 \mathrm{mg} \cdot \mathrm{L}^{-1}\right)$ (Table 1), such alterations may be explained by variations in the degree of silification of Synura echinulate scales. These alterations have also been reported to occur in vitro [95].

Synura mammillosa is a boreal species. In Synura mammillosa collected in the present study, some scales bore transverse ribs on the marginal part (Figure S5m) and some present a pattern of contradirectional ribs (Figure S51). The presence of such ornamentation on the scales of Synura mammillosa has been previously described in Aquitaine, France ([96]; Figure 52).

Spiniferomonas bourrellyi is a cosmopolitan species. Interestingly, we noted differences in the constructions and length of spines on the scales of this species collected in the present study. First, some samples possessed a 14-16.5- $\mu \mathrm{m}$-long spine with a coni- 
cal base and tapering toward the tip (Figure S3c). This type of spine was described by Green [97] in a new species of Spiniferomonas (Spiniferomonas andersonii Green). However, Nicholls [87] synonymised Spiniferomonas andersonii as Spiniferomonas bourrellyi. Second, in some samples, the spine was only $2.3-3.0 \mu \mathrm{m}$ long and possessed a saucer-shaped base (diameter, 2.2-2.5 $\mu \mathrm{m}$ ); this spine slightly widened at the base and then tapered toward the tip (Figure S3e). This type of spine has been described in samples collected from lakes in Connecticut [90] and the Labynkyr and Vorota lakes in Yakutia, Russia [48]. However, in Spiniferomonas bourrellyi collected from Olenyok in the present study, the spine was considerably smaller than that described previously.

\section{Discussion}

The considerable diversity (82 taxa) of silica-scaled chrysophytes in the Arctic rivers we investigated is comparable to the diversity in another Arctic region-the Bolshezemelskaya tundra of Russia (75 taxa) [22]. The species richness of silica-scaled chrysophytes is often high in waters with slightly acidic $\mathrm{pH}$ and low dissolved salt content, and in oligotrophic to mesotrophic waters with moderate content of dissolved humic compounds [98-101]. The geochemical context of the development of waters of the Bolshezemelskaya tundra is, however, considerably different. The waterbodies in this tundra are small lakes and ponds, that is, closed natural systems, with different levels of trophicity (both oligotrophic lakes and lakes with anthropogenic load), different acidic to alkaline $\mathrm{pH}$ values (5.5-8), and wide ranges of conductivity $\left(19-146 \mu \mathrm{S} \cdot \mathrm{cm}^{-1}\right)$ and temperature $\left(8-19.7^{\circ} \mathrm{C}\right)$ [22]. Waters of the rivers studied here showed comparable values of hydrochemical parameters (Table 2). The studied rivers of Yakutia, despite the increased phosphorus content, can be classified as oligotrophic, according to the classification proposed by Dodds et al. [102]. In oxygen/iron-rich waters, biologically available phosphorus accounts for only $8 \%$ of total phosphorus [102]. The $\mathrm{pH}$ and temperature at different stations range within 6.69-7.93 (neutral to slightly alkaline) and $12-17^{\circ} \mathrm{C}$, respectively. Likewise, a high species richness of silica-scaled chrysophytes (79 species) has been previously recorded in the Baikal Region with similar primary hydrochemical parameters $[13,103] . \mathrm{pH}$ is one of the major factors driving the development and distribution of silica-scaled chrysophytes [3]. In the present study, however, we obtained data that water $\mathrm{pH}$ at different stations, ranging from neutral to alkalescent or alkaline values, did not affect the species composition and richness of silica-scaled chrysophytes in the rivers of Yakutia. Summarising data obtained by the statistical analysis of 28 parameters of the habitat, the major factors governing species composition and the richness of silica-scaled chrysophytes were the provenance of the sample from a specific river; the distance of the sampling site from the estuary (i.e., the sea); water temperature, transparency, and colour; and concentration of carbon dioxide, magnesium ions, and total iron. The factor related to sampling from a specific river confirms the previously identified regional nature of the diversity of silica-scaled chrysophytes in northern reservoirs [40]. However, the mouths of the rivers we examined are located in the same region, and therefore these differences may have been driven by the diversity inherited by the rivers, which flow over a vast territory. Further, water pollution from surfactants and petroleum products have a considerable impact on species diversity. While settlements and anthropogenic factors other than poor navigation do not exist in the region, active development of the region and increased water pollution with surfactants and petroleum products can have a negative impact on the diversity of silica-scaled chrysophytes. The reaction of silica-scaled chrysophytes to changes in the concentration of magnesium and iron ions was intriguing, as this reaction was previously undetected. We have formerly discussed the importance of transparency and colour to the development of silica-scaled chrysophytes and phytoplankton in general [103-105]. Increases in water turbidity as a result of warming and the removal of terrigenous material by meltwater play significant roles in inhibiting the 
development of chrysophytes. Accordingly, we did not observe chrysophytes in rivers with high turbidity in this study.

The silica-scaled chrysophytes are known to produce different phenotypes of scales in response to different environmental conditions $[95,96,106]$. However, some species exhibit such phenotypic plasticity more often than others do. Previous geometric morphometric analyses have revealed the phenotypic plasticity of scales in Synura echinulate grown with different combinations of light, temperature, and $\mathrm{pH}$ [95]. Variations in the shapes of spine bases have been described in the Spiniferomonas bourrellii complex [87]. Despite the observed morphological differences in the structure of individual scales, taxonomically relevant traits of species remained intact. Therefore, such morphological alterations are a result of phenotypic plasticity, likely induced by one or many environmental factors.

Analysing the species composition of silica-scaled chrysophytes in other northern reservoirs located above the Arctic Circle on a time scale, we observed that cosmopolitans and widespread species predominate in the longitude group (Figure 3).

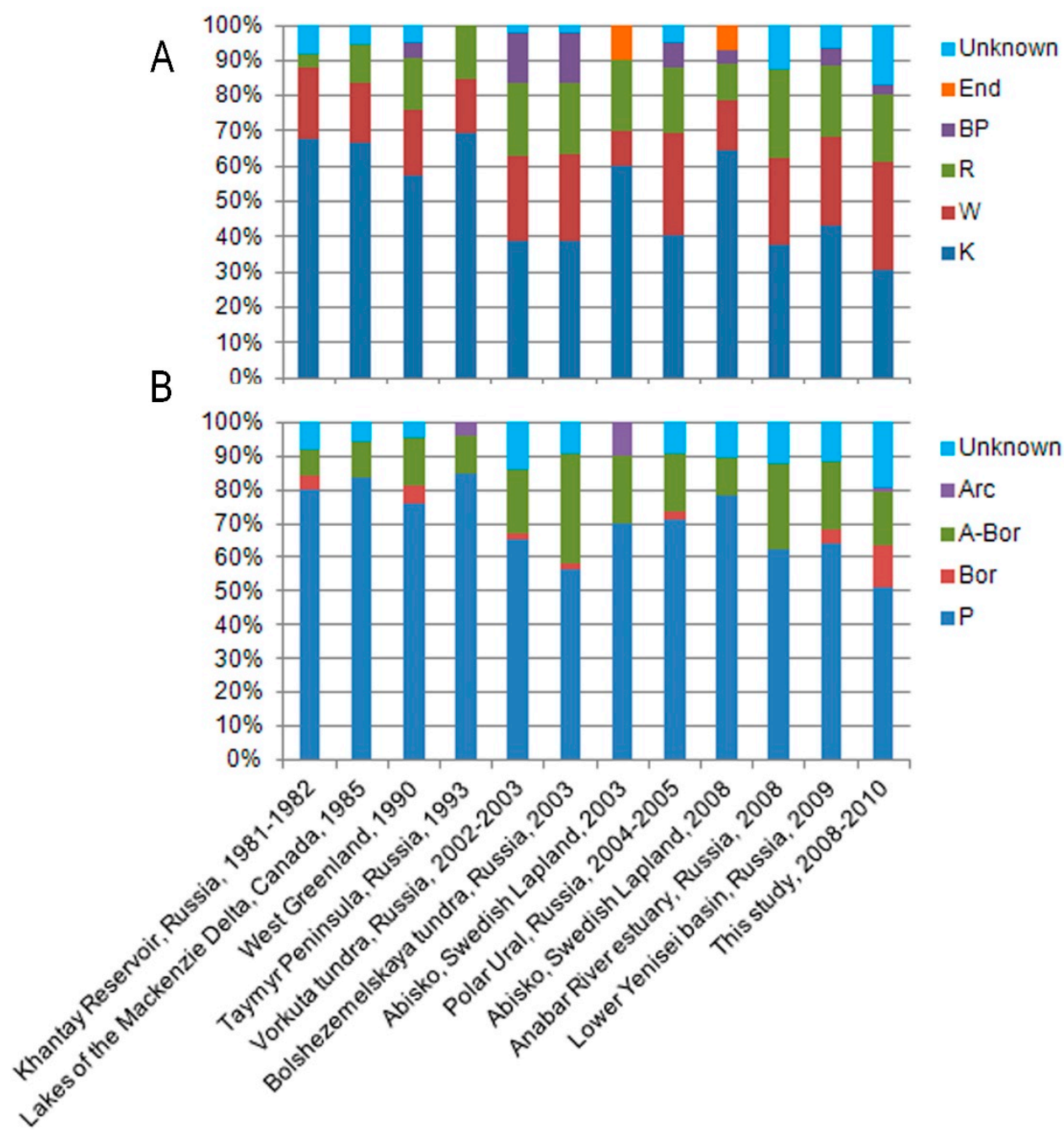

Figure 3. Comparison of species composition of silica-scaled chrysophytes by their grouping in the longitudinal (A) and latitudinal (B) groups (according to $[59,60,107]$ for waters north of the Arctic Circle from 1981 to 2010.

As such, the proportion of cosmopolitan species amongst all chrysophytes has changed from $68 \%$ in the Khantay Reservoir of Russia during 1981-1982 [28] to 30.5\% in the Arctic rivers of Yakutia in the present study. Simultaneously, however, the proportion of ubiquitous species has increased from $20 \%$ to $30.5 \%$. Species with scattered distribution, including rare species, became abundant during 1981-1982. The maximum number of bipolar species 
(14.3\%) has been recorded in the Vorkuta and Bolshezemelskaya tundra of Russia [22]. Synura obesa Němcova, Novakova \& Řezačova-Škaloudova remains restricted to Abisko in Swedish Lapland [19], which is the only species that has retained its endemicity amongst all other endemics. In the present study, for the first time since its description, we found Synura petersenii f. taymyrensis, which was suggested to be endemic to the Taymyr Peninsula [14]. Owing to a high number of potentially new species (17\%), the proportion of species with unidentified geographic characteristics was the highest in the present study.

Polyzonal species were predominant in the latitudinal group. The proportion of these species amongst all chrysophytes varies between $84.6 \%$ in the Taymyr Peninsula of Russia in 1993 [9] and 51.2\% in the Arctic rivers of Yakutia in the present study. The maximum number of Arctic-boreal species (32.7\%) was recorded in the Bolshezemelskaya tundra of Russia in 2003 [22], and the minimum (8\%) was recorded in the Khantay Reservoir of Russia [28]. We noted a decrease in the proportion of Arctic-boreal species and an increase in the proportion of boreal species of silica-scaled chrysophytes in the waters of the studied Arctic Region (Figure 3) compared with previously published data. Boreal species found in the studied rivers included Chrysosphaerella rotundata, Paraphysomonas cf. punctata ssp. simplicior, Paraphysomonas vulgaris Lepidochromonas eiffelii, Lepidochromonas elegantissima, Spiniferomonas takahashii, Synura curtispina f. reticulata, Synura glabra, and Synura mammillosa. In addition, two species, namely Synura petersenii f. taymyrensis and Synura obesa, can be considered Arctic. A high proportion of species with unidentified geographic characteristics $(14.2 \%)$ was recorded in the Vorkuta tundra of Russia during 2002-2003 [22] due to the high number of bipolar species. In the present study, this proportion was $19.5 \%$ due to the high number of potentially new species.

\section{Conclusions}

By establishing the biogeographic structure of silica-scaled chrysophyte communities in the mouths of rivers in the Arctic region of Yakutia, and comparing these with similar structures in other previously studied northern reservoirs, we detected in the temporal aspect a decrease in the proportion of cosmopolitans and an increase in the proportion of widespread species and those with scattered distribution. Specifically, we noted a decrease in the proportion of polyzonal species and an increase in the percentage of boreal and arcto-boreal species. This confirms our hypothesis that species have been introduced from temperate latitudes into Arctic reservoirs in the context of global climate change. The record level of species diversity of these microeukaryotes and the change in the structure of their communities reflect the beginning of manifestations of climate warming. The results of the study are important for the present and further monitoring of an untouched, environmentally sensitive area of the world, and are relevant for assessing the future consequences of local anthropogenic impacts and climate change.

Supplementary Materials: The following are available online at https:/ / www.mdpi.com/article / 10.3390/su132413768/s1, Table S1: Species composition of silica-scaled chrysophytes north of the Arctic Circle, Table S2: Raw data table for statistical analysis, Figure S1: Silica-scaled chrysophytes of the genera Chrysosphaerella, Clathromonas and Paraphysomonas identified in this study: a, b, Chrysosphaerella brevispina (site 4); c, d, Chrysosphaerella sp. (c site 5; d site 12); e, C. coronacircumspina (site 6); f, C. rotundata (site 5); g, C. longispina (site 4); h, Paraphysomonas sp. 1 (site 6); i, P. vulgaris (site 2); j, P. acuminata acuminata (site 4); k, Paraphysomonas sp. 2 (site 5); 1, P. bandaiensis (site 5); m, P. gladiata (site 12); n, P. uniformis hemiradia (site 6); o, P. cf. punctata ssp. simplicior (site 12); p, P. punctata

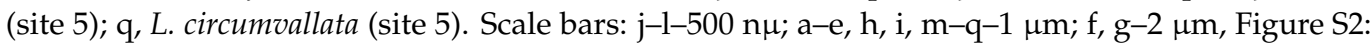
Silica-scaled chrysophytes of the genera Paraphysomonas and Lepidochromonas identified in this study: a-c, Paraphysomonas sp. 3 (a, b convex side of scale proximal, c distal side of scale (a site 4 ; b, c site 5)); d, Paraphysomonas sp. 4 (site 5); e, Lepidochromonas quadrispina (site 5); f, g, L. eiffelii (site 5); h, L. elegantissima (site 5); i, L. butcheri (site 5); j, L. diadernifera (site 5); k, L. undulata (site 5); 1, Lepidochromonas sp. 1 (site 5); m, L. subquadrangularis (site 5); n, Lepidochromonas sp. 2 (site 5); o, L.

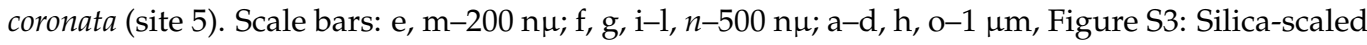
chrysophytes of the genus Spiniferomonas identified in this study: a, Spiniferomonas trioralis (site 7); b, 
S. trioralis f. cuspidata (site 4); c, e, S. bourrellyi (c site 5; e site 6); d, f, S. serrata (site 3); g, S. abei (site 5); h, S. cornuta (site 12); i, S. silverensis (site 2); j, S. conica (site 2); k, S. minuta (site 4); 1, S. takahashii (site 5). Scale bars: h-500 n $; 1-1 \mu \mathrm{m} ; \mathrm{a}-\mathrm{g}, \mathrm{i}-\mathrm{k}-2 \mu \mathrm{m}$, Figure S4: Silica-scaled chrysophytes of the genus Mallomonas identified in this study: a, Mallomonas acaroides (site 3); b, M. akrokomos (site 12); c, M. actinoloma (site 12); d, M. alata f. hualvensis (site 5); e, M. alpina (site 3); f, g, M. kuzminii (f site 3; g site 11); h, M. tonsurata (site 32); i, M. elongata (site 12); j, M. areolata (site 2); k, M. crassisquama (site 26); l, M. cratis (site 3); m, n, M. caudata (site 26); o, M. sf. pumilio (site 4); $p$, M. eoa (site 5). Scale bars: $b-1 \mu \mathrm{m} ; \mathrm{a}, \mathrm{c}-p-2 \mu \mathrm{m}$, Figure S5: Silica-scaled chrysophytes of the genera Mallomonas and Synura identified in this study: a, Mallomonas lychenensis (site 4); b, M. multiunca (site 2); c, d, M. punctifera (site 4); e, M. parvula (site 11); f, M. papillosa (site 4); g, M. striata (site 26); h, Synura uvella (site 26); i-k, S. echinulate (i site 12; j site 5; k site 32); 1, m, S. mammillosa (site 11); n, S. leptorhabda (site 12); o, Synura sp. 1 (site 12); p, S. nygaardii (site 3). Scale bars: e, $\mathrm{f}-1 \mu \mathrm{m}$; a-d, g-p-2 $\mu \mathrm{m}$, Figure S6: Silica-scaled chrysophytes of the genus Synura identified in this study: a, Synura sphagnicola (site 5); b, S. spinosa $\mathrm{f}$. longispina (site 30); c, S. spinosa (site 7); d, S. curtispina f. reticulata (site 3); e, S. curtispina (site 5); f, S. petersenii (site 11); g, h, S. borealis (site 2); i, Synura sp. 3 (site 4); j-1, S. americana (j site 2; k, 1 site 7); m, $\mathrm{n}, \mathrm{S}$. sf. cornuta (m site 1; n site 3); o-q, S. sf. vinlandica (site 7); r, s, S. conopea (r site 5; s site 31). Scale bars $2 \mu \mathrm{m}$, Figure S7: Silica-scaled chrysophytes of the genus Synura identified in this study: $a, b$, Synura sf. soroconopea (site 3); c, d, Synura sp. 2 (c site 9; d site 7); e, S. macropora (site 11); f, S. petersenii f. taymyrensis (site 5); g, S. praefracta (site 5); h, S. glabra (site 4), i-1, Synura sp. 4 (i site 4; g site 5; k, 1 site 11). Scale bars $2 \mu \mathrm{m}$.

Author Contributions: Conceptualisation, A.B. and Y.L.; data curation, V.G., O.G., A.B., Y.B. and A.F. writing-original draft preparation, A.B. and Y.L. All authors have read and agreed to the published version of the manuscript.

Funding: The work was conducted within the State Project 0297-2021-0023 `Vegetation of the permafrost taiga zone of Yakutia: biodiversity, environment-forming functions, protection and rational use' (Institute for Biological Problems of Cryolithozone, organisation and execution of expeditions, water chemistry analyses), and the State Project 0279-2021-0008 (Limnological Institute, Siberian Branch of the Russian Academy of Science, study of chrysophytes and statistical analyses).

Institutional Review Board Statement: Not applicable.

Informed Consent Statement: Not applicable.

Data Availability Statement: The data that support the findings of this study are available from the corresponding authors upon reasonable request.

Acknowledgments: The microscopy studies were performed in the Electron Microscopy Center of the Shared Research Facilities 'Ultramicroanalysis' of Limnological Institute (http:/ / www.lin.irk.ru/copp/).

Conflicts of Interest: The authors declare no conflict of interest.

\section{References}

1. Wujek, D.E. Freshwater silica-scaled heterotrophic protista: Heliozoa, thaumatomonad flagellates, amoebae, and bicosoecids, from the Lake Itasca Region, Minnesota. Minn. Acad. Sci. J. 2015, 79, 1-13.

2. Siver, P.A. The Biology of Mallomonas: Morphology, Taxonomy and Ecology; Kluwer Academic Publishers: Dordrecht, The Netherlands, 1991; p. 248.

3. Siver, P.A. Synurophyte algae. In Freshwater Algae of North America: Ecology and Classification, 2nd ed.; Academic Press: Boston, MA, USA, 2015; pp. 607-651.

4. Dürrschmidt, M.; Croome, R. Mallomonadaceae (Chrysophyceae) from Malaysia and Australia. Nord. J. Bot. 1985, 5, 285-298. [CrossRef]

5. Neustupa, J.; Řezáčová, M. The genus Mallomonas (Mallomonadales, Synurophyceae) in several Southeast Asian urban water bodies-The biogeographical implications. Nova Hedwig. 2007, 84, 249-259. [CrossRef]

6. Gusev, E.S.; Thant, N.T.H. Silica-scaled chrysophytes (Chrysophyceae and Synurophyceae) from Vietnam (Khanh Hoa and Quang Nam provinces). Nova Hedwig. 2011, 93, 191-199. [CrossRef]

7. Gusev, E.S.; Kapustin, D.A.; Martynenko, N.A.; Guseva, E.E.; Kulikovskiy, M.S. Mallomonas gusakovii sp. nov. (Chrysophyceae, Synurales), a new species from Phu Quoc Island, Vietnam. Phytotaxa 2019, 406, 199-205. [CrossRef]

8. Duff, K.E. Chrysophyte microfossils in arctic Siberian lakes. Chrysophytes: Progress and new horizons. Nova Hedwig. Beih. 1996, $114,253-263$. 
9. Kristiansen, J.; Düwel, L.; Wegeber, S. Silica-scaled chrysophytes from the Taymyr Peninsula, Northern Siberia. Nova Hedwig. 1997, 65, 337-351. [CrossRef]

10. Gusev, E.S.; Guseva, E.E.; Gabyshev, V.A. Taxonomic composition of silica-scaled chrysophytes in rivers and lakes of Yakutia and Magadanskaya oblast (Russia). Nova Hedwig. Beih. 2018, 147, 105-117. [CrossRef]

11. Hällfors, G.; Hällfors, S. Records of chrysophytes with siliceous scales (Mallomonadaceae and Paraphysomonadaceae) from Finnish inland waters. Flagellates in freshwater ecosystems. Hydrobiologia 1988, 161, 1-29. [CrossRef]

12. Němcová, Y.; Kreidlová, J.; Kosová, A.; Neustupa, J. Lakes and pools of Aquitaine region (France)—A biodiversity hotspot of Synurales in Europe. Nova Hedwig. 2012, 95, 1-24. [CrossRef] [PubMed]

13. Bessudova, A.Y.; Sorokovikova, L.M.; Tomberg, I.V.; Likhoshway, Y.V. Silica-scaled chrysophytes in large tributaries of Lake Baikal. Cryptogam. Algol. 2018, 39, 145-165. [CrossRef]

14. Siver, P.A. The synurophyceae. In Freshwater Algae of North America; Wehr, J., Sheath, B., Eds.; Academic Press: Thornwood, NY, USA, 2003; pp. 523-558.

15. Asmund, B.; Hilliard, D.K. Studies on Chrysophyceae from some ponds and lakes in Alaska. Hydrobiolology 1961, 7, 237-258. [CrossRef]

16. McKenzie, C.; Kling, H. Scale-bearing Chrysophyceae (Mallomonadaceae and Paraphysomonadaceae) from Mackenzie Delta area lakes, Northwest Territories, Canada. Nord. J. Bot. 1989, 9, 103-112. [CrossRef]

17. Kristiansen, J. Silica-scaled chrysophytes from west Greenland: Disko island and the Søndre Strømfjord region. Nord. J. Bot. 1992, 12, 525-536. [CrossRef]

18. Forsström, L.; Sorvari, S.; Korhola, A.; Rautio, M. Seasonality of phytoplankton in subarctic Lake Saanajärvi in NW Finnish Lapland. Pol. Biol. 2005, 28, 846-861. [CrossRef]

19. Němcová, Y.; Nováková, S.; Řezáčová-Škaloudová, M. Synura obesa sp. nov. (Synurophyceae) and other silica scaled chrysophytes from Abisko (Swedish Lapland). Nova Hedwig. 2008, 86, 243-254. [CrossRef]

20. Pichrtová, M.; Janatková, K.; Němcová, Y. Silica-scaled chrysophytes from Abisko (Swedish Lapland). Nord. J. Bot. 2011, 29, 112-118. [CrossRef]

21. Kristiansen, J. Flagellates from Finnish Lappland. Bot. Tidsskr. 1964, 59, 315-333.

22. Siver, P.A.; Voloshko, L.N.; Gavrilova, O.V.; Getsen, M.V. The scaled chrysophyte flora of the Bolshezemelskaya tundra (Russia). Nova Hedwig. Beih. 2005, 128, 125-150.

23. Voloshko, L.N. A new species of the genus Mallomonas (Chrysophyceae, Synurophyceae) from lakes of the Vorkuta tundra. Bot. Zhurn. 2012, 97, 1226-1234. (In Russian)

24. Voloshko, L.N. New taxa of the genus Mallomonas (Chrysophyceae, Synurophyceae) from lakes of the Polar Urals. Bot. Zhurn. 2009, 94, 1068-1076. (In Russian)

25. Voloshko, L.N. The chrysophycean algae from glacial lakes of Polar Ural (Russia). Nova Hedwig. Beih. 2010, 136, 191-211. [CrossRef]

26. Firsova, A.D.; Bessudova, A.Y.; Sorokovikova, L.M.; Tomberg, I.V.; Likhoshway, Y.V. The diversity of chrysophycean algae in an arctic zone of river and sea water mixing, Russia. Am. J. Plant Sci. 2015, 6, 2439-2452. [CrossRef]

27. Bessudova, A.Y.; Bukin, Y.S.; Sorokovikova, L.M.; Firsova, A.D.; Tomberg, I.V. Silica-scaled Chrysophytes in small lakes of the Lower Yenisei basin, the Arctic. Nova Hedwig. 2018, 107, 315-336. [CrossRef]

28. Balonov, I.M.; Kuzmina, A.E. Golden algae. In Proceedings of Limnological Institute, RAS of the Academy of Sciences of USSRP; Votintsev, K.K., Ed.; Hydrochemical and Hydrobiological Studies of the Khantay Reservoir, Nauka, Siberian Branch: Novosibirsk, Russia, 1986; pp. 59-70. (In Russian)

29. Wolfe, A.P.; Siver, P.A. A hypothesis linking chrysophyte microfossils to lake carbon dynamics on ecological and evolutionary time scales. Glob. Planetary Chang. 2013, 111, 189-198. [CrossRef]

30. Mushet, G.R.; Laird, K.R.; Das, B.; Hesjedal, B.; Leavitt, P.R.; Scott, K.A.; Simpson, G.L.; Wissel, B.; Wolfe, J.D.; Cumming, B.F. Regional climate changes drive increased scaled-chrysophyte abundance in lakesdownwind of Athabasca Oil Sands nitrogen emissions. J. Paleolimnol. 2017, 58, 419-435. [CrossRef]

31. Schindler, D.W. The cumulative effects of climate warming and other human stresses on Canadian freshwaters in the new millennium. Can. J. Fish. Aquat. Sci. 2001, 58, 18-29. [CrossRef]

32. Paterson, A.M.; Winter, J.G.; Nicholls, K.H.; Clarks, B.J.; Ramcharan, C.W.; Yan, N.D.; Somers, K.M. Long-term changes in phytoplankton composition in seven Canadian Sheet lakes in response to multiple anthropogenic stressors. Can. J. Fish. Aquat. Sci. 2008, 65, 846-861. [CrossRef]

33. Rühland, K.M.; Paterson, A.M.; Smol, J.P. Hemispheric-scale patterns of climate-related shifts in planktonic diatoms from North American and European lakes. Glob. Chang. Biol. 2008, 14, 2740-2754. [CrossRef]

34. Field, C.B.; Barros, V.R.; Dokken, D.J.; Mach, K.J.; Mastrandrea, M.D.; Bilir, T.E.; Chatterjee, M.; Ebi, K.L.; Estrada, Y.O.; Genova, R.C.; et al. Part A: Global and Sectoral Aspects. Contribution of Working Group II to the Fifth Assessment Report of the Intergovernmental Panel on Climate Change. In Climate Change 2014: Impacts, Adaptation, and Vulnerability; Cambridge University Press: New York, NY, USA, 2014; pp. 1-32.

35. Magnuson, J.J.; Robertson, D.M.; Benson, B.J.; Wynne, R.H.; Livingstone, D.M.; Arai, R.A.; Barry, R.G.; Card, V.; Kuusisto, E.; Granin, N.G.; et al. Historical trends in lake and river ice cover in the Northern Hemisphere. Science 2000, 289, 1743-1746. [CrossRef] 
36. Vuglinsky, V.; Valatin, D. Changes in ice cover duration and maximum ice thickness for rivers and lakes in the Asian part of Russia. Nat. Res. 2018, 9, 73-87. [CrossRef]

37. Korneva, L.G. Recent invasion of planktonic diatom algae in the Volga River basin and their causes. Inland Wat. Boil. 2007, 1, 30-39. (In Russian)

38. Korneva, L.G. Invasions of alien species of planktonic microalgae into the fresh waters of Holarctic (Review). Russ. J. Biol. Invasions 2014, 1, 9-37. [CrossRef]

39. Korneva, L.G. Phytoplankton of Reservoirs of the Volga River Basin; Kostroma: Dom Pechati, Russia, 2015; p. 284. (In Russian)

40. Bessudova, A.; Bukin, Y.; Likhoshway, Y. Dispersal of silica-scaled Chrysophytes in Northern Water bodies. Diversity 2021, 13, 284. [CrossRef]

41. Kirillov, F.N.; Labutina, T.M. Biology of Vilyuy Reservoir; Nauka: Novosibirks, Russia, 1979; p. 272. (In Russian)

42. Vasilyeva, I.I.; Remigaylo, P.A. Algae of Vilyuy Reservoir; Yakutia Scientific Center USSR Academy of Sciences Publishing House: Yakutsk, Russia, 1982; p. 115. (In Russian)

43. Remigaylo, P.A. Phytoplankton of the Vilyuy River and Vilyuy Reservoir. Ph.D. Thesis, Novosibirsk University, Novosibirsk, Russia, 1995; p. 16. (In Russian)

44. Vasilyeva-Kralina, I.I.; Remigaylo, P.A.; Gabushev, V.A.; Pshennikova, E.V.; Ivanova, A.P.; Kopyrina, L.I. Algae. In Diversity of Plants of Yakutia; Publishing House SB RAS: Novpsibirsk, Russia, 2005; pp. 150-272. (In Russian)

45. Gabyshev, V.A.; Gabysheva, O.I. Phytoplankton of Big Rivers of Yakutia and Adjacent Territories of East Siberia; Korneva, L.G., Ed.; SibAK Press: Novosibirks, Russia, 2018; p. 414. (In Russian)

46. Gabyshev, V.A.; Tsarenko, P.M.; Ivanova, A.P. Diversity and features of the spatial structure of algal communities of water bodies and watercourses in the Lena River estuary. Inland Water Biol. 2019, 12, 1-9. [CrossRef]

47. Gabyshev, V.A.; Tsarenko, P.M.; Ivanova, A.P. Algae of mouth area of Lena River. In The Biological Resources of the Ust-Lensky Nature Reserve: Fungi, Algae, Vegetation, Fishes, Birds, Muskoxen; Science: Novosibirsk, Russia, 2019; pp. 14-35.

48. Bessudova, A.Y.; Tomberg, I.V.; Firsova, A.D.; Kopyrina, L.I.; Likhoshway, Y.V. Silica-scaled chrysophytes in lakes Labynkyr and Vorota of the Sakha (Yakutia) Republic, Russia. Nova Hedwig. Beih. 2019, 148, 35-48. [CrossRef]

49. Izyumenko, S.A. (Ed.) Climate of the Yakut ASSR. In Atlas; Gidrometeoizdat: Leningrad, Russia, 1968; p. 33. (In Russian)

50. Chistyakov, G.E. River Water Resources of Yakutia; Nauka: Moskow, Russia, 1964; p. 255. (In Russian)

51. Chistyakov, G.E. Hydropower Resources of Drainage Basins of the Yana River; Chistyakov, G.E., Nogovitsyn, D.D., Yakushev, M.V., Eds.; Nauka: Moskow, Russia, 1970; p. 214. (In Russian)

52. Resources of Inland Waters of the USSR. Lena-Indigirka Region; Gidrometeoizdat: Leningrad, Russia, 1972; Volume 17, p. 651. (In Russian)

53. Resources of Inland Waters of the USSR. North-East; Gidrometeoizdat: Leningrad, Russia, 1966; Volume 19, p. 602. (In Russian)

54. Guidelines for Collection and Processing of Samples at Hydrobiological Studies of Fresh Waters; Nauka: Leningrad, Russia, $1981 ;$ p. 32. (In Russian)

55. Wiebe, P.H.; Benfield, M.C. From the Hensen net toward four-dimensional biological oceanography. Prog. Oceanogr. 2003, 56, 7-136. [CrossRef]

56. Alyokin, O.A.; Semyonov, A.D.; Skopintsev, B.A. Guidelines for Chemical Analysis of Inland Waters; Gidrometeoizdat: Leningrad, Russia, 1973; p. 269. (In Russian)

57. Semyonov, A.D. Guidelines for Chemical Analysis of Inland Water; Gidrometeizdat: Leningrad, Russia, 1977; p. 540. (In Russian)

58. Sokolova, S.A.; Anisova, S.N. (Eds.) List of Commercial Fishing Standards: Maximum Allowable Concentrations (MACs) and Safe Reference Levels of Contaminants in Commercial Fishing Waters; VNIRO: Moskow, Russia, 1999; p. 304. (In Russian)

59. Kristiansen, J. Dispersal and biogeography of silica-scaled chrysophytes. Biodiv. Conserv. 2008, 17, 419-426. [CrossRef]

60. Voloshko, L.N. Golden Algae of Waters of North Russia; Komarov Botanical Institute of the Russian Academy of Sciences: SanktPetersburg, Russia, 2017; p. 378. (In Russian)

61. Anderson, M.J. A new method for non-parametric multivariate analysis of variance. Aust. Ecol. 2001, 26, 32-46. [CrossRef]

62. Oksanen, J.; Blanchet, F.G.; Kindt, R.; Legendre, P.; Minchin, P.R.; O'Hara, R.B.; Simpson, G.L.; Solymos, P.; Stevens, M.H.H.; Wagner, H. Vegan: Community Ecology Package, 2014. R Package Version 2.2-0. Available online: http://CRAN.Rproject.org/ package=vegan (accessed on 13 August 2021).

63. Oksanen, J. Vegan: An Introduction to Ordination. 2015. Available online: http://cran.r-project.org/web/packages/vegan/ vignettes/introvegan.pdf (accessed on 13 August 2021).

64. Škaloud, P.; Škaloudová, M.; Jadrná, I.; Bestová, H.; Pusztai, M.; Kapustin, D.A.; Siver, P.A. Comparing morphological and molecular estimates of species diversity in the freshwater genus Synura (stramenopiles): A model for understanding diversity of eukaryotic microorganisms. J. Phycol. 2020, 56, 574-591. [CrossRef] [PubMed]

65. Jo, B.Y.; Kim, J.I.; Škaloud, P.; Siver, P.A.; Shin, W. Multigene phylogeny of Synura (Synurophyceae) and descriptions of four new species based on morphological and DNA evidence. Europ. J. Phycol. 2016, 51, 413-430. [CrossRef]

66. Škaloudová, M.; Škaloud, P. A new species of Chrysosphaerella (Chrysophyceae: Chromulinales), Chrysosphaerella rotundata, sp. nov., from Finland. Phytotaxa 2013, 130, 34-42. [CrossRef]

67. Dürrschmidt, M. Studies on scale-bearing Chrysophyceae from the Giessen area, Federal Republic of Germany. Nord. J. Bot. 1984, 4, 123-143. [CrossRef] 
68. Thomsen, H.A.; Zimmermann, B.; Moestrup, O.; Kristiansen, J. Some new freshwater species of Paraphysomonas (Chrysophyceae). Nord. J. Bot. 1981, 1, 559-581. [CrossRef]

69. Kristiansen, J. On the species of Paraphysomonas (Chrysophyceae) in some Greek lakes. Nova Hedwig. 1983, 38, 65-72.

70. Kling, H.J.; Kristiansen, J. Scale-bearing Chrysophyceae (Mallomonadaceae) from Central and Northern Canada. Nord. J. Bot. 1983, 3, 269-290. [CrossRef]

71. Nicholls, K.H. Five Paraphysomonas species (Chrysophyceae) new to North America, with notes on three other rarely reported species. Can. J. Bot. 1985, 63, 1208-1212. [CrossRef]

72. Hickel, B.; Maass, I. Scaled chrysophytes, including heterotrophic nanoflagellates from the lake district in Holstein, northern Germany. Nova Hedwig. Beih. 1989, 95, 233-257.

73. Wujek, D.E.; O'Kelly, C.J. Silica-scaled Chrysophyceae (Mallomonadaceae and Paraphysomonadaceae) from New Zealand freshwaters. N. Z. J. Bot. 1992, 30, 405-414. [CrossRef]

74. Ikävalko, J. Observations on silica-scaled flagellates (Chrysophyceae and Synurophyceae) in the brackish water of Pojo Bay, SW coast of Finland. Ann. Bot. Fen. 1994, 31, 1-27.

75. Hällfors, G. Checklist of Baltic Sea Phytoplankton species (including some heterotrophic protistan groups). Balt. Sea Environ. Proc. 2004, 95, 1-208.

76. Preisig, H.R.; Hibberd, D.J. Ultrastructure and taxonomy of Paraphysomonas (Chrysophyceae) and related genera 2. Nord. J. Bot. 1982, 2, 601-638. [CrossRef]

77. Dürrschmidt, M. Studies on the Chrysophyceae from South Chilean inland waters by means of scanning and transmission electron microscopy, II. Archiv für Hydrobiologie 63. Algol. Stud. 1982, 63, 121-163.

78. Nicholls, K.H. Eight chrysophyceae new to North America. Phycologia 1984, 23, 213-221. [CrossRef]

79. Barreto, S. The silica-scaled chrysophyte flora of Hungary. Nova Hedwig. Beih. 2005, 128, 11-41.

80. Pichrtová, M.; Němcová, Y.; Škaloud, P.; Rott, E. Silica-scaled chrysophytes from North Tyrol (Austria) including a description of Mallomonas tirolensis sp. nov. Nova Hedwig. Beih. 2013, 142, 69-85.

81. Wei, Y.-X.; Yuan, X.-P. Studies on silica-scaled chrysophytes from the Daxinganling mountains and Wudalianchi Lake regions, China. Nova Hedwig. 2015, 101, 299-312. [CrossRef]

82. Nielsen, Y. Spiniferomonas abrupta, sp. nov. and some rare species of Synurophyceae and Chrysophyceae, not formerly recorded from Denmark. Nord. J. Bot. 1994, 14, 473-480. [CrossRef]

83. Ikävalko, J.; Thomsen, H.A. Scale-covered and loricate flagellates (Chrysophyceae and Synurophyceae) from Baltic Sea ice. Nova Hedwig. Beih. 1994, 114, 147-160.

84. Ikävalko, J. Contribution to the flora of silica-scaled flagellates in Mikkeli, central Finland. Nova Hedwig. 1994, 58, 475-505.

85. Finlay, B.J.; Clarke, K.J. Apparent global ubiquity of species in the protist genus Paraphysomonas. Protist 1999, 150, 419-430. [CrossRef]

86. Takahashi, E. Studies on genera Mallomonas and Synura, and other plankton in fresh water with the electron microscope. VII. New genus Spiniferomonas of the Synuraceae (Chrysophyceae). Bot. Mag. Tok. 1973, 86, 75-88. [CrossRef]

87. Nicholls, K.H. Spiniferomonas (Chrysophyceae) in Ontario lakes including a revision and descriptions of two new species. Can. J. Bot. 1981, 59, 107-117. [CrossRef]

88. Jacobsen, V.A. Scale-bearing Chrysophyceae (Mallomonadaceae and Paraphysomonadaceae) from West Greenland. Nord. J. Bot. 1985, 5, 381-398. [CrossRef]

89. Nicholls, K.H. Descriptions of Spiniferomonas silverensis sp. nov. and S. minuta sp. nov. and an assessment of form variation in their closest relative, S. trioralis (Chrysophyceae). Can. J. Bot. 1984, 62, 2329-2335. [CrossRef]

90. Siver, P.A. The distribution and ecology of Spiniferomonas (Chrysophyceae) in Connecticut (U.S.A). Nord. J. Bot. 1988, 8, $205-212$. [CrossRef]

91. Němcová, Y.; Pusztai, M.; Škaloudová, M.; Neustupa, J. Silica-scaled chrysophytes (Stramenopiles, Ochrophyta) along a salinity gradient: A case study from the Gulf of Bothnia western shore (Northern Europe). Hydrobiologia 2016, 764, 187-197. [CrossRef]

92. Siver, P.A.; Wujek, D.E. Scaled Chrysophyceae and Synurophyceae from Florida, U.S.A.: VI. Observations on the flora from waterbodies in the Ocala National Forest. Nova Hedwig. 1999, 68, 75-92. [CrossRef]

93. Bessudova, A.Y.; Domysheva, V.M.; Firsova, A.D.; Likhoshway, Y.V. Silica-scaled chryso phytes of Lake Baikal. Acta Biol. Sib. 2017, 3, 47-56. [CrossRef]

94. Babich, D.B.; Korotaev, V.N.; Magritsky, D.V.; Mikhailov, V.N. Lower Indigirka: Estuarine and Riverbed Processes; GEOS: Moskow, Russia, 2001; p. 202.

95. Němcová, Y.; Neustupa, J.; Kvíderová, J.; Řezáčová-Škaloudová, M. Morphological plasticity of silica scales of Synura echinulate (Synurophyceae) in crossed gradients of light and temperature-A geometric morphometric approach. Nova Hedwig. Beih. 2010, 136, 21-32. [CrossRef]

96. Němcová, Y.; Pichrtová, M. Shape dynamics of silica scales (Chrysophyceae, Stramenopiles) associated with pH. Fottea Olomouc 2012, 12, 281-291. [CrossRef]

97. Green, R.B. A new species of Spiniferomonas (Chrysophyceae) from an Alberta Lake. Can. J. Bot. 1979, 57, 557-560. [CrossRef]

98. Eloranta, P. Biogeography of chrysophytes in Finnish lakes. In Chrysophyte Algae: Ecology, Phylogeny and Development; Sandgren, C.D., Smol, J.P., Kristiansen, J., Eds.; Cambridge University Press: Cambridge, UK, 1995; pp. 214-231. [CrossRef] 
99. Siver, P.A. The distribution of chrysophytes along environmental gradients: Their use as biologicalindicators. In Chrysophyte Algae; Sandgren, C.D., Smol, J.P., Kristiansen, J., Eds.; Cambridge University Press: Cambridge, UK, 1995; pp. $232-268$.

100. Kristiansen, J. Golden Algae: A Biology of Chrysophytes; A.R.G. Gantner Verlag: Königstein, Germany, $2005 ;$ p. 167.

101. Siver, P.A.; Lott, A.M. The scaled chrysophyte flora in freshwater ponds and lakes from Newfoundland, Canada, and their relationship to environmental variables. Cryptogam. Algol. 2017, 38, 325-347. [CrossRef]

102. Dodds, W.K.; Jones, J.R.; Welch, E.B. Suggested classification for stream trophic state: Distributions of temperate stream types by chlorophyll, total nitrogen and Phosphorus. Water Res. 1998, 32, 1455-1462. [CrossRef]

103. Bessudova, A.Y.; Sorokovikova, L.M.; Sinyukovich, V.N.; Firsova, A.D.; Tomberg, I.V.; Likhoshway, Y.V. Effects of water levels on species diversity of silica-scaled chrysophytes in large tributaries of Lake Baikal. Acta Biol. Sib. 2020, 95, 1-24. [CrossRef]

104. Rojo, C.; Mesquita-Joanes, F.; Monrós, J.S.; Armengol, J.; Sasa, M.; Bonilla, F.; Rueda, R.; Benavent-Corai, J.; Piculo, R.; Seguraet, M.M. Hydrology affects environmental and spatial structuring of microalgal metacommunities in tropical Pacific coast wetlands. PLoS ONE 2016, 11, e0149505. [CrossRef] [PubMed]

105. Talbot, C.J.; Bennett, K.M.; Cassell, K.; Hanes, D.M.; Minor Hans, E.C.; Paerl, H.; Raymond, P.A.; Vargas, R.; Vidon, P.G.; Wollheim, W.; et al. The impact of flooding on aquatic ecosystem services. Biogeochemistry 2018, 141, 439-461. [CrossRef] [PubMed]

106. Řezáčová-Škaloudová, M.; Neustupa, J.; Němcová, Y. Effect of temperature on the variability of silicate structures in Mallomonas kalinae and Synura curtispina (Synurophyceae). Nova Hedwig. Beih. 2010, 136, 55-69. [CrossRef]

107. Kristiansen, J. Cosmopolitan chrysophytes. Syst. Geogr. Plants 2000, 70, 291-300. [CrossRef] 